\title{
Article \\ Impact of sex and pathophysiology on optimal drug choice in hypertensive rats: Quantitative insights for precision medicine
}

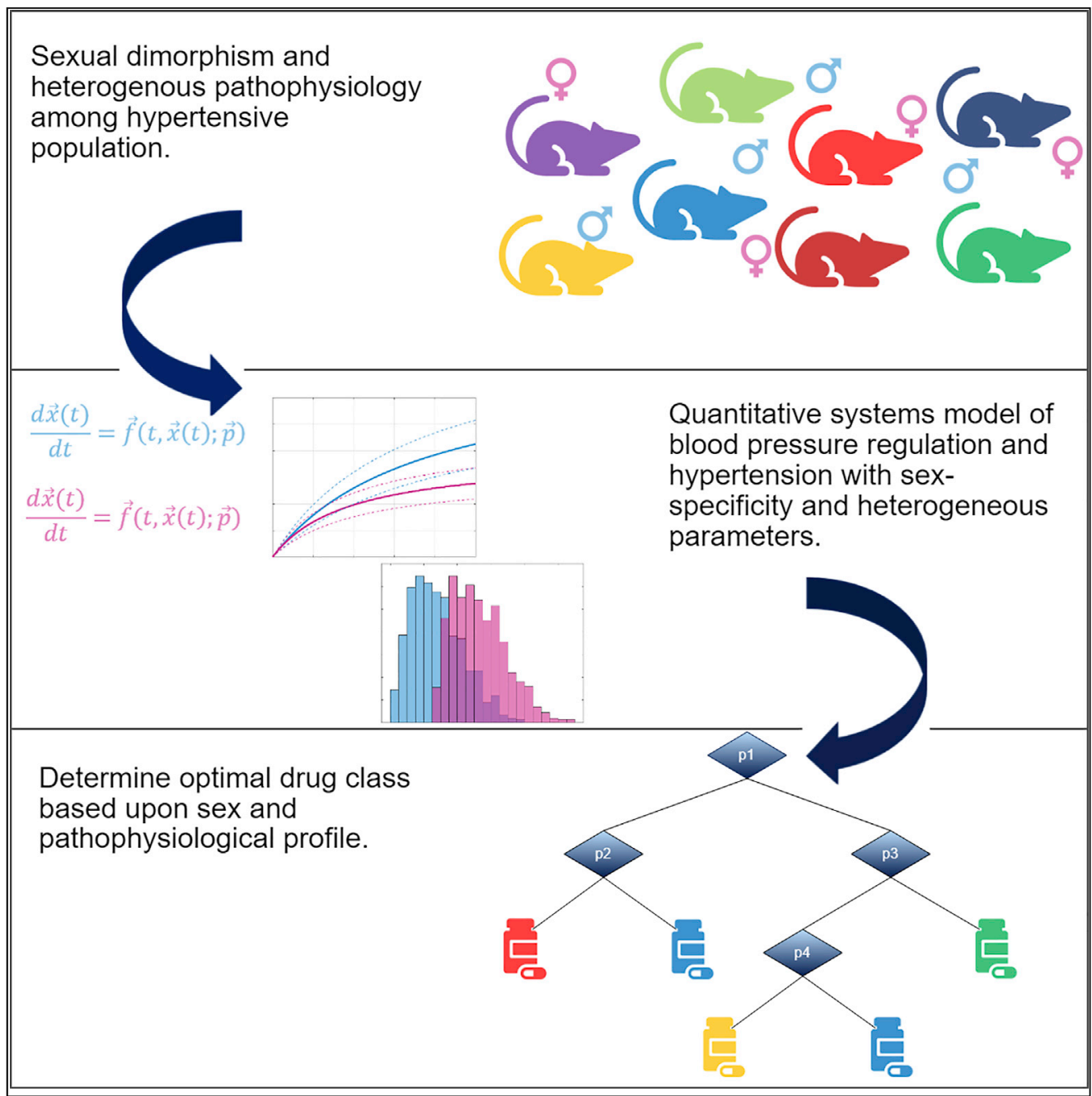

Sameed Ahmed, Jennifer C. Sullivan, Anita T.

Layton

sameed.ahmed@uwaterloo.ca

Highlights

A quantitative systems pharmacology model was developed for

hypertensive rats

The model accounts for heterogeneity in sex and pathophysiology

Optimal drug classes for reducing blood pressure were determined

Sex and pathophysiological profile are promising areas for precision medicine 


\title{
Article \\ Impact of sex and pathophysiology on optimal drug choice in hypertensive rats: Quantitative insights for precision medicine
}

\author{
Sameed Ahmed, ${ }^{1,4, *}$ Jennifer C. Sullivan, ${ }^{2}$ and Anita T. Layton ${ }^{1,3}$
}

\begin{abstract}
SUMMARY
Less than half of all hypertensive patients receiving treatment are successful in normalizing their blood pressure. Despite the complexity and heterogeneity of hypertension, the current antihypertensive guidelines are not tailored to the individual patient. As a step toward individualized treatment, we develop a quantitative systems pharmacology model of blood pressure regulation in the spontaneously hypertensive rat (SHR) and generate sex-specific virtual populations of SHRs to account for the heterogeneity between the sexes and within the pathophysiology of hypertension. We then used the mechanistic model integrated with machine learning tools to study how variability in these mechanisms leads to differential responses in rodents to the four primary classes of antihypertensive drugs. We found that both the sex and the pathophysiological profile of the individual play a major role in the response to hypertensive treatments. These results provide insight into potential areas to apply precision medicine in human primary hypertension.
\end{abstract}

\section{INTRODUCTION}

Hypertension is a major preventable risk factor for cardiovascular disease and stroke, affecting about $46 \%$ of adults in the United States (Virani Salim et al., 2020). Despite various treatment efforts, only about half of all hypertensive patients receiving treatment achieve adequate blood pressure control (Ritchey et al., 2018). This low success rate may be attributable, in part, to the characterization of most (90\%-95\%) of the hypertensive patients by primary, or essential, hypertension, which is a heterogeneous disease with multiple underlying pathophysiological mechanisms (Oparil et al., 2018). Compounding the issue is that sex differences exist in the regulation of blood pressure, prevalence and etiology of hypertension, and response to therapy (Kalibala et al., 2020; Reckelhoff, 2018). Meanwhile, although the eighth report of the Joint National Committee on the prevention, detection, evaluation, and treatment of high blood pressure provides antihypertensive guidelines that are broadly specified for certain ages, ethnicities, and comorbidities, they are not tailored to the patient. In particular, the guidelines are not distinguished for either the pathophysiological profile or the sex of the patient (James et al., 2014).

Several organs and systems are involved in regulating blood pressure, including the kidney, nervous system, and endocrine system. These systems collectively detect variations in blood pressure and respond accordingly to restore blood pressure homeostasis. This is accomplished by regulating arterial resistances through changing vascular muscle tone or by regulating body fluid levels through changing sodium reabsorption along the nephron. Primary hypertension results from the aberrance in several of these regulators, which makes it difficult to treat.

Quantitative systems pharmacology (QSP) models are useful for understanding complex mechanisms, testing hypotheses, and guiding treatment (Clegg and Mac Gabhann, 2015; Gibson et al., 2018). The seminal quantitative model for blood pressure regulation was developed by Guyton et al. (1972) and has since been updated and expanded by several researchers (Ahmed and Layton, 2020; Hallow and Gebremichael, 2017a; Hallow et al., 2014; Hester et al., 2011; Karaaslan et al., 2005; Leete and Layton, 2019). In previous studies, we developed the first sex-specific quantitative model for the renin-angiotensin system (RAS) (Leete et al., 2018); we then incorporated the RAS model into a sex-specific model blood pressure
${ }^{1}$ Department of Applied Mathematics, University of Waterloo, Waterloo, ON N2L 3G1, Canada

2Department of Physiology, Medical College of Georgia, Augusta University, Augusta, GA 30912, USA

${ }^{3}$ Department of Biology,

Cheriton School of Computer Science, and School of Pharmacology, University of Waterloo, Waterloo, ON, N2L 3G1, Canada

${ }^{4}$ Lead contact

*Correspondence: sameed.ahmed@uwaterloo. ca

https://doi.org/10.1016/j.isci. 2021.102341 


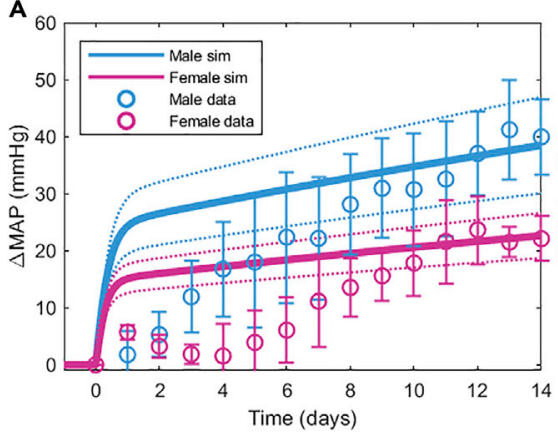

B

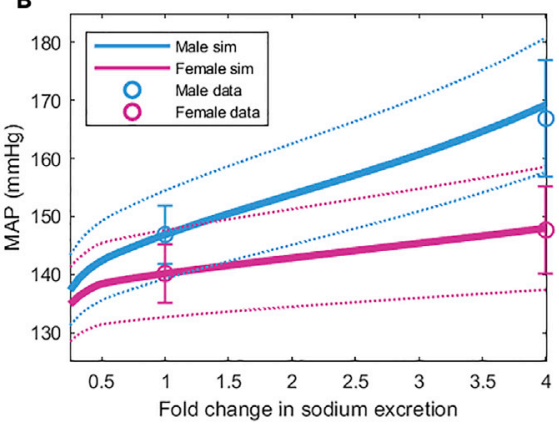

Figure 1. Model simulated results across the virtual population along with experimental data under experimental scenarios used to estimate pathophysiological parameters

Model simulations are given by the lines, and experimental data are given by the points. Solid lines are the average value, and dotted lines are the $95 \%$ confidence interval for the model simulation. Error bars are the $95 \%$ confidence interval (A) or range $(B)$ for the experimental data.

(A) Mean arterial pressure (MAP) time course when angiotensin (Ang) II is infused at day 0. Experimental data are from Sullivan et al. (2010).

(B) MAP steady-state values in response to sodium loading. Experimental data are from Brinson et al. (2014) and Wyss et al (1987, 1994).

See also Figure S2.

regulation in the rat and used the resulting model to understand sex-specific physiological mechanisms responsible for sexual dimorphisms in blood pressure regulation (Ahmed and Layton, 2020). We now utilized this model to develop the first model of primary hypertension based upon the spontaneously hypertensive rat (SHR). We created a virtual population to represent the genetic diversity in the pathophysiology of hypertension and then used the model to study the mechanisms attributable for differential responses to antihypertensive treatment among male and female SHR populations.

\section{RESULTS}

\section{Formation of the virtual spontaneously hypertensive rat individual and population}

By perturbing the selected parameters that represent the etiology of hypertension in the SHR and fitting these parameters to experimental data, we create a virtual SHR. This means that key physiological variables are within range, and the model simulations under perturbation of Ang II infusion and sodium loading match experimental data (Sullivan et al. [2010] for the former and Brinson et al. [2014], Wyss et al [1987, 1994] for the latter). Model simulation results alongside experimental data across male and female virtual populations are plotted in Figure 1A under Ang II infusion and in Figure 1B under sodium loading. There is good agreement between the calibrated virtual population and the corresponding experimental data. Following this procedure, we create male and female virtual populations consisting of 1,000 individuals for each sex. Posterior distributions for the fitted pathophysiological parameters are given in Figure 2. The simulated baseline normotensive values of key physiological variables as well as the mean and standard deviation across the hypertensive virtual populations are given in Table 1. Also provided are the experimental ranges for the variables, which are the physiological constraints that are imposed when estimating the parameters. All simulated key physiological variables are within experimental range. When compared with the normotensive virtual individual, most hypertensive virtual individuals are characterized by an elevated vascular resistance (Figures 2A-2C), with males having a greater proportion of individuals with elevated arterial resistance (Figure 2A) and females having a greater proportion of individuals with elevated venous resistance (Figure 2B). Afferent arteriolar resistance has a similar distribution in virtual males and females. Most hypertensive virtual individuals also have greater renin secretion, aldosterone secretion, renal sympathetic nerve activity, and antidiuretic hormone secretion (Figures 2D-2G) than normotensive virtual individuals. An important point to note is that while imposing physiological constraints on the variables (Table 1), we still observe a wide spread in the pathophysiological parameters (Figure 2). Full details on developing the hypertensive model and generating the virtual population are provided in transparent methods. For a complete list of all model variable and parameter values, see Table S1. 


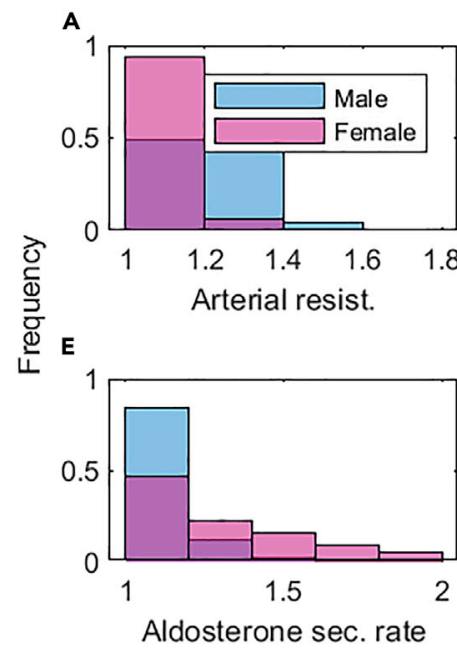

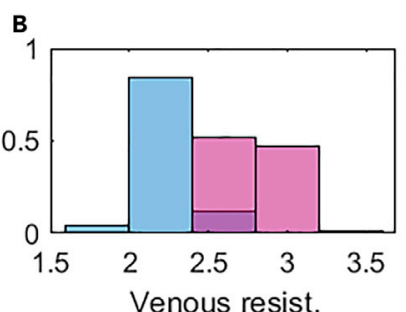

$\mathbf{F}$

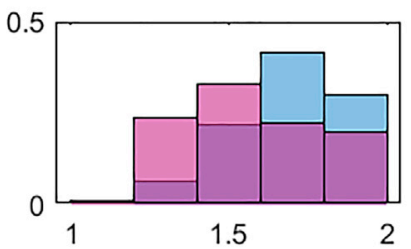

Renal sympathetic nerve act.
C

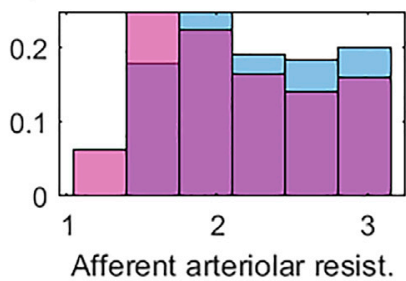

G

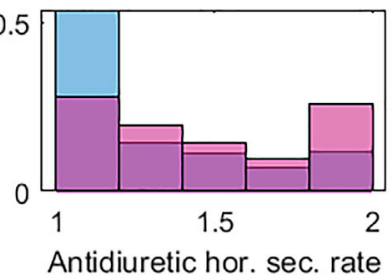

D

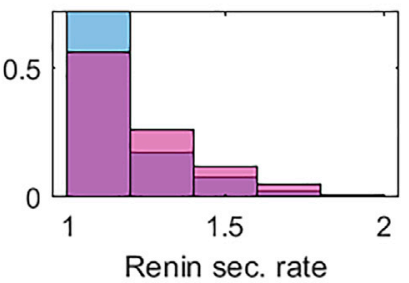

H

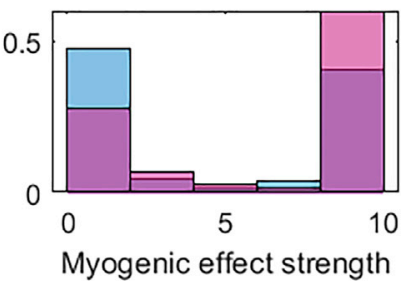

Fold Change

Figure 2. Distribution of parameters involved in the etiology of hypertension in male and female virtual populations

Values are plotted as fold change from baseline. The perturbed parameters are those related to $(A)$ arterial resistance; $(B)$ venous resistance; $(C)$ afferent arteriolar resistance; (D) renin secretion rate; $(E)$ aldosterone secretion rate; $(F)$ renal sympathetic nerve activity; $(G)$ antidiuretic hormone secretion rate; $(H)$ strength of myogenic effect. See also Figures S1 and S2.

\section{Calibration and validation of antihypertensive drugs simulations \\ Angiotensin-converting enzyme inhibitor and angiotensin receptor blocker}

We simulate the administration of angiotensin-converting enzyme (ACE) inhibitor (ACEi) by inhibiting the reaction rate of ACE converting Ang I into Ang II. By choosing $96 \%$ inhibition of $C_{A C E}$, we observe on average a $4.13 \pm 0.07$-fold increase in plasma renin activity (PRA) in males, which is within range of experimental data on male SHRs given ACEis captopril, benazerpril, and perindopril (Antonaccio et al, 1979, 1980; Bunkenburg et al., 1991; Unger et al., 1986). Model calibration and validation with experimental data for drug simulations are summarized in Table 2. As a result, simulated mean arterial pressure (MAP) decreases significantly by $19.2 \% \pm 1.6 \%$ in males, which is consistent with experimental results (Table 2) (Antonaccio et al., 1979; Bunkenburg et al., 1991; Richer et al., 1987). Females have a similar simulated MAP decrease of $19.9 \% \pm 5.2 \%$. We observe a large decrease in simulated glomerular filtration rate (GFR) in males and females of $32.6 \% \pm 2.5 \%$ and $23.4 \% \pm 2.8 \%$, respectively. GFR response to ACEi in males is consistent with experimental results (Francischetti et al., 1998). The inhibition response curve for the entire inhibition range of $C_{A C E}$ is shown in Figure $3 A$, where we simulate the steady-state MAP response corresponding to the level of inhibition. We observe a greater decrease in MAP for greater inhibition levels, which is indicative of the thresholding of various autoregulatory mechanisms involved in blood pressure regulation. Note that this model simulation is an inhibition response curve. Therefore we do not observe the typical curve shape seen in dose-response curves, whereby the curve levels off for greater doses due to a saturation in response.

Similarly, we administer an angiotensin receptor blocker (ARB) to the model by inhibiting the binding rate of Ang II to its type 1 receptor (AT1R). By choosing 94\% inhibition of $C_{A T 1 R}$, we observe on average a $6.50 \pm$ 0.20 -fold increase in PRA in males, which is within range of experimental data on male SHRs given ARBs candesartan and losartan (Table 2) (Bunkenburg et al., 1991; Kohara et al., 1993; Mizuno et al., 1992a, 1992b). We observe similar qualitative results as with administration of ACEi, but with a larger magnitude. MAP decrease in males is consistent with experimental results (Table 2) (Bunkenburg et al., 1991; Kohara et al., 1993; Wong et al., 1991). Simulated MAP decreases by $23.4 \% \pm 2.0 \%$ in males and $27.3 \% \pm 5.2 \%$ in females, and simulated GFR decreases by $42.5 \% \pm 2.8 \%$ in males and $32.5 \% \pm 3.3 \%$ in females. Model prediction in males for the decrease in GFR in response to ARB agrees qualitatively with experimental results but overestimates the effect (Fenoy et al., 1991). The simulated inhibition response curve for the entire inhibition range of $C_{A T 1 R}$ is shown in Figure $3 \mathrm{~B}$.

Administering ACEi or ARB results in a decrease in Ang II bound to AT1R, which has a multitude of downstream effects, ultimately resulting in a reduction in MAP due to a reduction in body fluids. This reduction in 


\begin{tabular}{|c|c|c|c|c|c|}
\hline Variable & Units & M, F NTN & M, F HTN & M, F Exp. Range & Reference \\
\hline Mean arterial pressure & $\mathrm{mmHg}$ & 103,103 & $147 \pm 4,140 \pm 4$ & {$[143,153],[133,143]$} & $\begin{array}{l}\text { (Freeman et al., 1975; Smith and } \\
\text { Hutchins, 1979; Sullivan et al., 2010) }\end{array}$ \\
\hline Cardiac output & $\mathrm{ml} / \mathrm{min}$ & $54.6,34.8$ & $56.3 \pm 1.3,35.6 \pm 1.0$ & {$[51.9,57.3],[33.1,36.5]$} & (Badyal et al., 2003) \\
\hline Total peripheral resistance & $\mathrm{mmHg} /(\mathrm{mL} / \mathrm{min})$ & $1.89,2.96$ & $2.61 \pm 0.07,3.93 \pm 0.07$ & {$[2.50,2.95],[3.64,4.32]$} & (Badyal et al., 2003) \\
\hline Renal blood flow & $\mathrm{ml} / \mathrm{min}$ & $13.1,8.35$ & $13.2 \pm 0.1,8.46 \pm 0.05$ & {$[12.4,13.8],[7.93,8.77]$} & (Arendshorst and Beierwaltes, 1979) \\
\hline Renal vascular resistance & $\mathrm{mmHg} /(\mathrm{mL} / \mathrm{min})$ & $7.87,12.3$ & $11.2 \pm 0.3,16.6 \pm 0.5$ & {$[10.4,12.3],[15.2,18.0]$} & (Arendshorst and Beierwaltes, 1979) \\
\hline Afferent arteriolar resistance & $\mathrm{mmHg} /(\mathrm{mL} / \mathrm{min})$ & $2.99,4.68$ & $6.29 \pm 0.34,8.95 \pm 0.46$ & {$[5.28,7.66],[7.18,10.7]$} & (Arendshorst and Beierwaltes, 1979) \\
\hline Efferent arteriolar resistance & $\mathrm{mmHg} /(\mathrm{mL} / \mathrm{min})$ & $4.88,7.64$ & $4.88 \pm 0.02,7.61 \pm 0.04$ & {$[4.64,5.12],[7.26,8.02]$} & (Arendshorst and Beierwaltes, 1979) \\
\hline Glomerular filtration rate & $\mathrm{ml} / \mathrm{min}$ & $2.44,1.67$ & $2.47 \pm 0.06,1.73 \pm 0.02$ & {$[2.32,2.56],[1.59,1.75]$} & (Arendshorst and Beierwaltes, 1979) \\
\hline Urine flow & $\mathrm{ml} / \mathrm{min}$ & $\begin{array}{l}0.0300 \\
0.0300\end{array}$ & $\begin{array}{l}0.0304 \pm 0.0005 \\
0.0300 \pm 0.0001\end{array}$ & $\begin{array}{l}{[0.0285,0.0315]} \\
{[0.0285,0.0315]}\end{array}$ & (Arendshorst and Beierwaltes, 1979) \\
\hline Blood volume & $\mathrm{Ml}$ & $15.0,12.0$ & $18.3 \pm 0.2,15.8 \pm 0.1$ &,-- & (Beierwaltes et al., 1982) \\
\hline Plasma $\mathrm{Na}^{+}$concentration & $\mu \mathrm{Eq} / \mathrm{min}$ & 143,147 & $143 \pm 1,146 \pm 1$ & {$[140,146],[144,150]$} & (Freeman et al., 1975) \\
\hline
\end{tabular}

Normotensive column is the model-simulated normotensive values. Hypertensive column is the model-simulated value of the mean \pm standard deviation among the hypertensive virtual population. Exp. Range column, if provided, is the physiological constraint from experimental data imposed when estimating the parameters for the SHR. Reference column provides the reference for the experimental data from where the range is determined.

body fluids is achieved through an increase in GFR and a decrease in sodium, and thus water, reabsorption along the nephron. Mechanisms explaining the drop in MAP are plotted in Figures 3C-3F for representative male and female virtual individuals who are administered ACEi at day 0 (see Figure $\mathrm{S} 1$ for the physiological systems connections). An increase in GFR is caused by the vasodilation of the renal vasculature such that the efferent arteriolar resistance to renal vascular resistance ratio increases (Figure 3C; see Equations S17-S21). Note that although the vasodilatory effect of decreasing AT1R-bound Ang II causes an increase in GFR, the net change of administering ACEi is a decrease in GFR. This is because the resulting decrease in MAP lowers the glomerular hydrostatic pressure, which in turn lowers GFR in greater magnitude (results not shown). A decrease in sodium reabsorption along the nephron occurs along the proximal segment, due to a decrease in AT1R-bound Ang II, and along the distal and collecting segments, due to a decrease in aldosterone (Figures 3D and 3E). Mechanisms for a drop in MAP after administering ARB are similar.

\section{Calcium channel blocker}

We simulate the administration of calcium channel blocker (CCB) by reducing the baseline afferent, efferent, and systemic arterial resistances by $85 \%, 57 \%$, and $57 \%$, respectively. This results in a net decrease of $70.5 \% \pm 1.0 \%$ in the afferent arteriolar resistance in males, which is within range of the in vivo experimental data reported for administering nifedipine in male SHRs (Table 2) (Messing et al., 1991). As a result, simulated MAP decreases by $16.1 \% \pm 1.6 \%$ in males, which is consistent with experimental results (Table 2) (Messing et al., 1991). Females have a greater decrease in simulated MAP of $19.6 \% \pm 3.5 \%$. Simulated GFR increases in males and females by $3.15 \% \pm 0.21 \%$ and $8.87 \% \pm 1.11 \%$, respectively. Model prediction in males for the increase in GFR in response to CCB agrees qualitatively with experimental results but underestimates the effect (Johns, 1988). The simulated inhibition response curve for the entire vasodilation range of the afferent arteriole is shown in Figure 4A.

Administering CCB results in a reduction in MAP due to reduction in arterial resistance and body fluids. It also causes an increase in GFR due to the greater effect on the afferent arteriole than the efferent arteriole, thereby increasing the efferent arteriolar resistance to renal vascular resistance ratio (Figure 4B). Mechanisms explaining the drop in MAP are plotted in Figures 4B-4F for representative male and female virtual individuals who are administered a CCB at day 0 . Although cardiac output increases due to a reduction in the resistance to venous return (Figure $4 \mathrm{E}$ ), the net effect is a reduction in MAP due to the larger decrease in total peripheral resistance (Figure 4F; see Equations S3-S5).

\section{Thiazide diuretic}

We simulate the administration of a thiazide diuretic (TZD) by decreasing the fractional amount of sodium along the distal segment of the nephron by $50 \%$ and increasing the amount of renin secretion by $100 \%$. This 


\begin{tabular}{|c|c|c|c|c|c|c|c|}
\hline Drug & Effect & Calibration & Exp. Range & Reference & Validation & Exp. Range & Reference \\
\hline ACEi & $96 \%$ & $\begin{array}{l}\text { PRA increase } \\
4.13 \pm 0.07 \text {-fold }\end{array}$ & {$[4,10]$} & $\begin{array}{l}\text { (Antonaccio et al., 1979; } \\
\text { Bunkenburg et al., 1991; } \\
\text { Unger et al., 1986) }\end{array}$ & $\begin{array}{l}\text { MAP decrease } \\
19.2 \pm 1.6 \%\end{array}$ & {$[13,36]$} & $\begin{array}{l}\text { (Antonaccio et al., 1979; } \\
\text { Bunkenburg et al., 1991; } \\
\text { Richer et al., 1987) }\end{array}$ \\
\hline ARB & $94 \%$ & $\begin{array}{l}\text { PRA increase } \\
6.50 \pm 0.20 \text {-fold }\end{array}$ & {$[6,14]$} & $\begin{array}{l}\text { (Bunkenburg et al., 1991; } \\
\text { Kohara et al., 1993; } \\
\text { Mizuno et al., 1992b) }\end{array}$ & $\begin{array}{l}\text { MAP decrease } \\
23.4 \pm 2.0 \%\end{array}$ & {$[14,27]$} & $\begin{array}{l}\text { (Bunkenburg et al., 1991; } \\
\text { Kohara et al., 1993; } \\
\text { Wong et al., 1991) }\end{array}$ \\
\hline $\mathrm{CCB}$ & $85 \%$ & $\begin{array}{l}\text { AAR decrease } \\
70.5 \pm 1.0 \%\end{array}$ & {$[50,80]$} & (Messing et al., 1991) & $\begin{array}{l}\text { MAP decrease } \\
16.1 \pm 1.6 \%\end{array}$ & {$[13,23]$} & (Messing et al., 1991) \\
\hline TZD & $50 \%, 100 \%$ & $\begin{array}{l}{\left[\mathrm{Na}^{+}\right] \text {decrease }} \\
0.88 \pm 0.20 \% \\
\text { PRA increase } \\
2.07 \pm 0.08 \text {-fold }\end{array}$ & $\begin{array}{l}{[0.2,1.2]} \\
{[2.2,3.2]}\end{array}$ & $\begin{array}{l}\text { (Yonetani et al., 1987) } \\
\text { (Lai et al., 1982; } \\
\text { Mougenot et al., 2005) }\end{array}$ & $\begin{array}{l}\text { MAP decrease } \\
7.72 \pm 0.86 \%\end{array}$ & {$[5.5,8.6]$} & $\begin{array}{l}\text { (Lai et al., 1982; Mougenot } \\
\text { et al., 2005) }\end{array}$ \\
\hline
\end{tabular}

Exp., experimental; ACEi, angiotensin-converting enzyme inhibitor; ARB, angiotensin receptor blocker; PRA, plasma renin activity; MAP, mean arterial pressure. Calibration and validation results are from model simulations, whereas the experimental range is determined from experimental data in the corresponding references.

results in a $0.88 \% \pm 0.20 \%$ decrease in plasma sodium concentration and a $2.07 \pm 0.08$-fold increase in plasma renin activity in males, which is within range of experimental data reported for hydrochlorothiazide administered in male SHRs (Table 2) (Lai et al., 1982; Mougenot et al., 2005; Yonetani et al., 1987). As a result, simulated MAP decreases by $7.72 \% \pm 0.86 \%$ in males, which is consistent with experimental results (Table 2) (Lai et al., 1982; Mougenot et al., 2005; Yonetani et al., 1987). Females have a much larger decrease in simulated MAP of $17.7 \% \pm 3.5 \%$ (see explanation below). Simulated GFR decreases in males and females by $7.15 \% \pm 1.55 \%$ and $23.0 \% \pm 1.1 \%$, respectively. GFR response to TZD in males is consistent with experimental results (Komatsu et al., 1996). The simulated inhibition response curve for the entire inhibition range of the distal tubule fractional reabsorption is shown in Figure 5A.

Administration of a TZD results in a reduction in MAP due to a reduction in body fluids. Mechanisms explaining the drop in MAP are plotted in Figures 5B-5E for representative male and female virtual individuals who are administered TZD at day 0 . The natriuresis and diuresis effects of TZD are acute, lasting about half a day, after which steady-state urine flow is slightly lower than the pre-drug value due to equilibration with a lower water intake (Figure 5C; see Equations S83 and S84). Initially, cardiac output decreases significantly due to fluid loss and total peripheral resistance increases slightly due to the autoregulatory feedback of a reduced MAP. After about half a day, total peripheral resistance gradually decreases, whereas cardiac output gradually increases, equilibrating at a new set point that sustains the chronic reduction in MAP (Figures 5D and 5E).

\section{Males and females respond differently to the different drug classes}

Angiotensin-converting enzyme inhibitor and angiotensin receptor blocker

Male and female virtual SHRs both experience a large reduction in MAP after administration of ACEi and ARB. For sufficiently large doses, the reduction in simulated MAP is larger in females than in males, despite the fact that females have a lower baseline MAP in hypertension (140 [F] versus 147 [M] mmHg; Table 1). Model simulations attribute this dimorphism in response to ACEi to two reasons. Females have a higher post-drug to pre-drug relative GFR when compared with males (i.e., a smaller decrease after drug administration; explained below). Additionally, sex differences are known in kidney function (Ahmed et al., 2019; Layton and Sullivan, 2018). In particular, when compared with males, females have a load shift of fractional sodium reabsorption from the proximal segment to the distal and collecting segments (Ahmed and Layton, 2020; Hu et al, 2019, 2020; Li et al., 2018; Veiras et al., 2017). And the latter two segments are more greatly impacted by the reduction in fractional sodium, and consequentially water, reabsorption due to the reduction in aldosterone concentration (Figures 3D and 3E; see Equations S39, S40, S44, and S47). Together, these two effects allow for a greater excretion of body fluids in females than in males (Figure 3F). The sexual dimorphism is greater in response to ARB than ACEi due to the additional effect of Ang II bound to its type 2 receptor (AT2R). In response to ACEi, Ang II bound to AT2R decreases, whereas in response to ARB, 
A

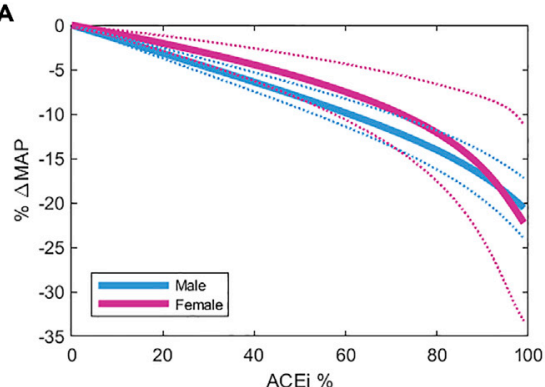

C

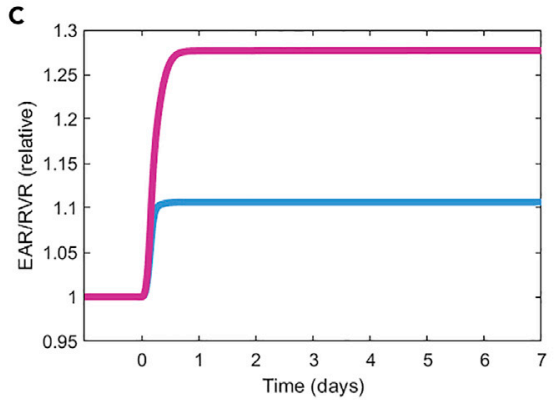

E

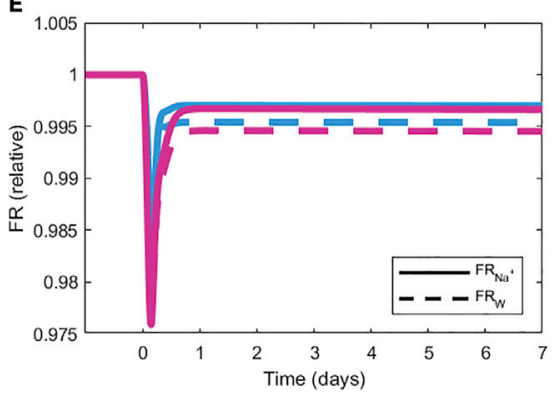

B

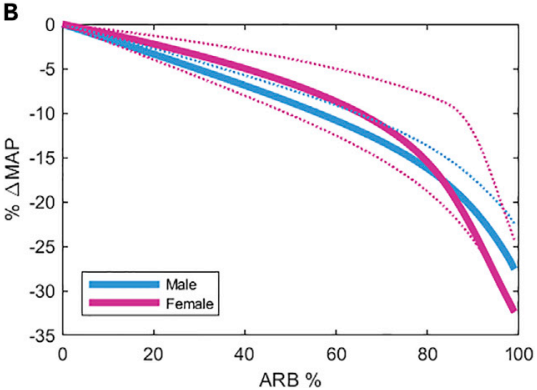

D

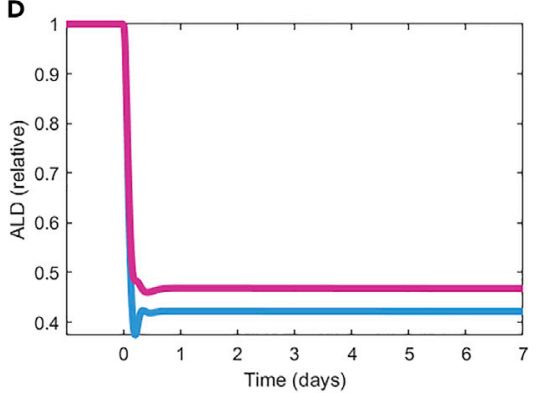

$\mathbf{F}$

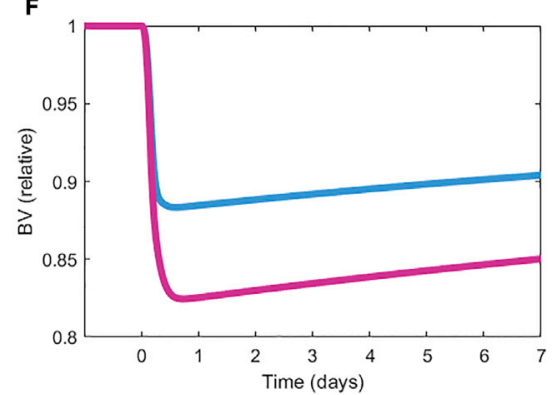

Figure 3. Model simulated response to angiotensin-converting enzyme inhibitor (ACEi) and angiotensin receptor blocker (ARB)

( $A$ and $B$ ) Steady-state mean arterial pressure (MAP) inhibition response to varying inhibition levels of the rate constant after administering (A) ACEi and (B) ARB. MAP is shown as relative percent change. Solid lines are the average value. Dotted lines are the $95 \%$ confidence interval.

(C-F) Time varying mechanisms explaining the drop in MAP after ACEi is administered at a $96 \%$ inhibition of the rate constant at day 0 in representative virtual individuals. All values are plotted as relative change from baseline. Mechanisms explaining the drop in MAP after ARB administration are similar. (C) Ratio of efferent arteriolar (EAR) to renal vascular resistance (RVR). (D) Aldosterone concentration (ALD). (E) Total fractional amount of sodium (FR $\left.\mathrm{NA}_{+}\right)$and water (FRW) reabsorbed in the kidney. (F) Blood volume (BV).

See also Figure S1.

AT2R-bound Ang II increases. This results in vasoconstriction and vasodilation, respectively, of the afferent arteriole in females only (Ahmed and Layton, 2020) (see Equations S22 and S29). A greater simulated MAP reduction in female SHRs at sufficiently high doses is consistent with experimental findings with ACEi (Dalpiaz et al., 2013; Gong et al., 1994) and ARB (Rodrigues et al., 2006; Zimmerman et al., 2014).

After administration of $A C E i$ or $A R B$, despite the larger decrease in MAP $(-19.2 \% \pm 1.6 \%$ [M] versus $-19.9 \% \pm 5.2 \%[F]$ and $-23.4 \% \pm 2.0 \%[\mathrm{M}]$ versus $-27.3 \% \pm 5.2 \%$ [F] for ACEi and ARB, respectively) in virtual females than in virtual males, females have a smaller decrease in simulated GFR $(-32.6 \% \pm 2.5 \%$ $[\mathrm{M}]$ versus $-23.4 \% \pm 2.8 \%[\mathrm{~F}]$ and $-42.5 \% \pm 2.8 \%[\mathrm{M}]$ versus $-32.5 \pm 3.3 \%[\mathrm{~F}]$ for ACEi and ARB, respectively). GFR decrease is due to a decrease in glomerular hydrostatic pressure. As aforementioned, this decrease is mitigated by the increase in the efferent arteriolar to renal vascular resistance ratio (Figure $3 \mathrm{C}$ ), which is due to a greater vasodilation of the afferent arteriole than the efferent arteriole. There are several 
A

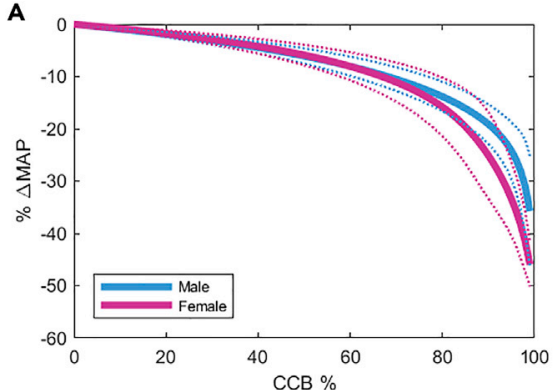

C

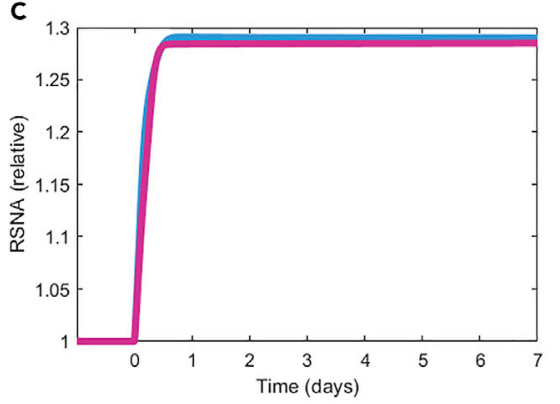

E

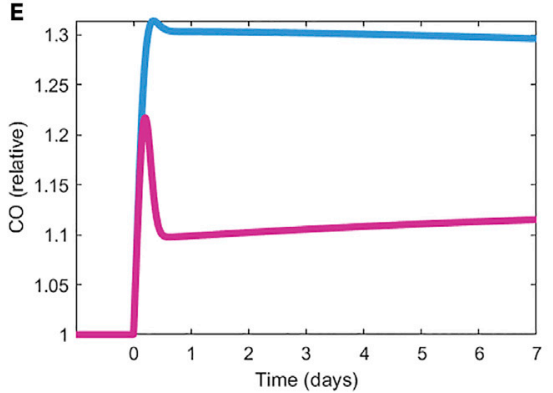

B

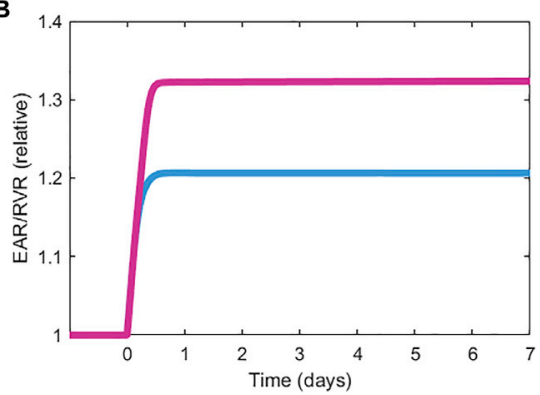

D

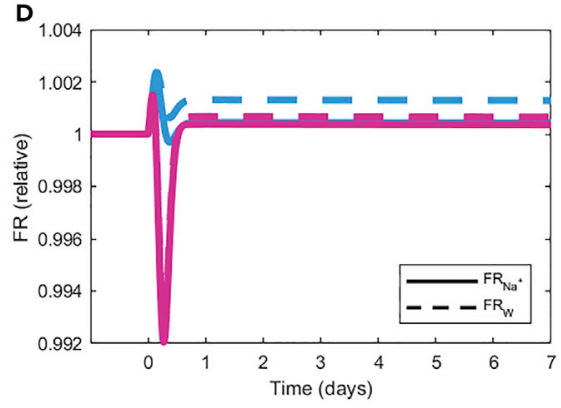

F

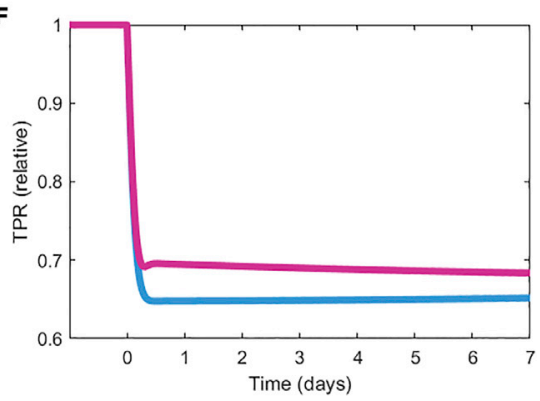

Figure 4. Model simulated response to calcium channel blocker (ССB)

(A) Steady-state mean arterial pressure (MAP) inhibition response to varying vasodilation levels of the arteries after administering CCB. MAP is shown as relative percent change. Solid lines are the average value. Dotted lines are the $95 \%$ confidence interval.

(B-F) Time varying mechanisms explaining the drop in MAP after CCB administered at an $85 \%$ vasodilation of the arteries at day 0 in representative virtual individuals. All values are plotted as relative change from baseline. (B) Ratio of efferent arteriolar (EAR) to renal vascular resistance (RVR). (C) Renal sympathetic nerve activity (RSNA). (D) Total fractional amount of sodium ( $F R_{N A_{+}}$) and water ( $F R_{W}$ ) reabsorbed in the kidney. (E) Cardiac output (CO). (F) Total peripheral resistance (TPR). See also Figure S1.

physiological mechanisms at play that affect the afferent arteriolar resistance after administration of ACEi/ $A R B$, two of which are the most significant, as suggested by model simulations, in causing the afferent arteriole in females to vasodilate more than that in males. The decrease in MAP causes renal sympathetic nerve activity (RSNA) to increase, which in turn increases the afferent arteriolar resistance. As females have a less excitatory RSNA (Ahmed and Layton, 2020) (see Equation S80), this increase is attenuated in females. Additionally, as females have a sodium load shift to segments downstream of the proximal segment, their macula densa functions at a higher setpoint by sensing more sodium at baseline. As ACEi/ARB decrease the amount of sodium flowing through macula densa, the decrease is greater in females. This causes a greater reduction in tubuloglomerular feedback signal in the female, which decreases the afferent arteriolar resistance.

\section{Calcium channel blocker}

Male and female virtual SHRs both experience a large reduction in MAP after administration of a CCB. Again, despite females having a lower baseline MAP (less hypertensive), the reduction is larger in females 
A

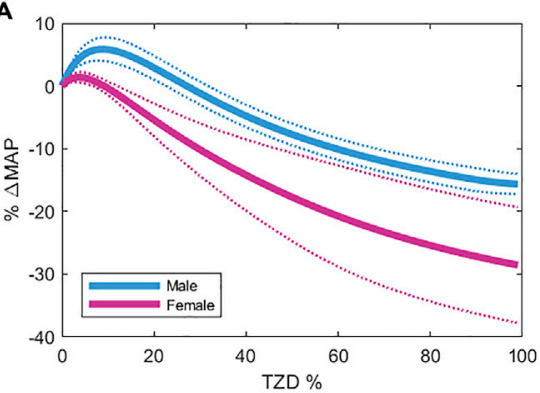

C

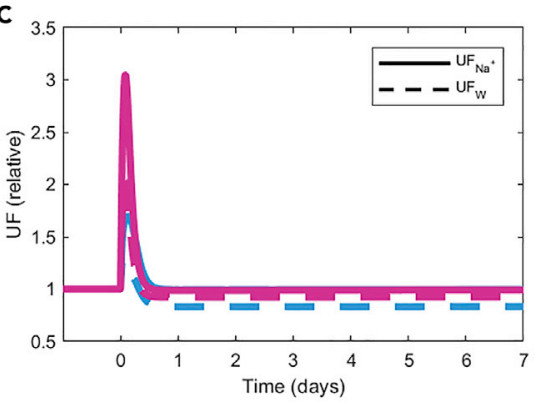

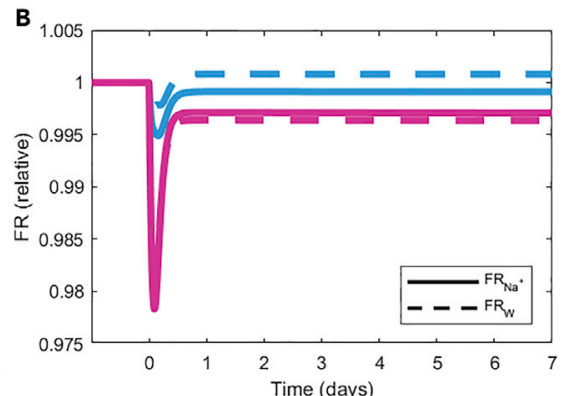

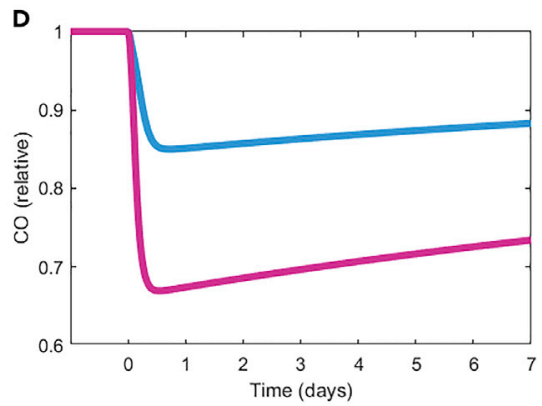

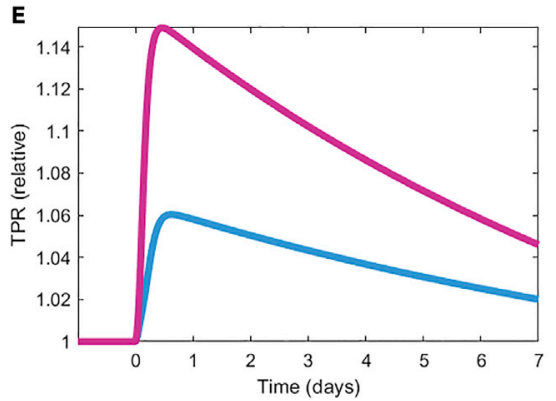

Figure 5. Model simulated response to thiazide diuretic (TZD)

(A) Steady-state mean arterial pressure (MAP) inhibition response to varying inhibition levels of fractional sodium reabsorption in the distal segment after administering TZD. MAP is shown as relative percent change. Solid lines are the average value. Dotted lines are the $95 \%$ confidence interval.

(B-E) Time varying mechanisms explaining the drop in MAP after TZD administered at a 50\% inhibition of distal segment fractional sodium reabsorption and a 100\% activation of renin secretion at day 0 in representative virtual individuals. All values are plotted as relative change from baseline. (B) Total fractional amount of sodium (FR $\mathrm{NA}_{+}$) and water (FR reabsorbed in the kidney. (C) Urine $\left(U F_{W}\right)$ and urine sodium (UF $\left.\mathrm{Na}_{+}\right)$flow rate. (D) Cardiac output (CO). (E) Total peripheral resistance (TPR).

See also Figure S1.

than in males $(-16.1 \% \pm 1.6 \%[\mathrm{M}]$ versus $-19.6 \% \pm 3.5 \%[\mathrm{~F}])$ for sufficiently high doses (greater than $\sim 65 \%$ vasodilation, Figure 5A). Model simulation results provide reasons similar to those with ACEi/ARB. Females have a lesser increase in simulated GFR $(+8.87 \% \pm 1.11 \%[\mathrm{M}]$ versus $+3.15 \% \pm 0.21 \%[\mathrm{~F}]$, explained below), as well as a greater decrease in body fluids due to a greater decrease in the total fractional amount of sodium and water reabsorbed by the kidney (Figure 4D). The latter is attributed to the sodium load shift from proximal segments to distal and collecting segments in the nephron in females, when compared with males.

After administration of a CCB, GFR increases due to an increase in the efferent arteriolar to renal vascular resistance ratio. This increase is greater in females (Figure $4 \mathrm{~B}$ ), due to the vasoconstrictive effect of RSNA on the afferent arteriole (see Figure S1 for the physiological systems connections), which is greater stimulated in males (see Equation S80). This can be seen in Figure 4C, where males have a slightly greater stimulated RSNA than females, despite have a lesser decrease in MAP. However, the greater decrease in MAP in females mitigates this effect on GFR, resulting in males having a greater increase in GFR. 


\section{Thiazide diuretic}

Female virtual SHRs experience a much larger reduction in MAP in response to TZD than virtual male SHRs $(-7.72 \% \pm 0.86 \%[\mathrm{M}]$ versus $-17.7 \% \pm 3.5 \%[\mathrm{~F}])$. This is due to the load shift in sodium and water reabsorption in the nephron of females away from the proximal segment toward the distal and collecting segments. As TZDs inhibit sodium-chloride cotransporter activity along the distal convoluted tubule, the resulting effect is much greater in females than males. This is consistent with experimental results in which female SHRs excrete much more sodium and fluid than males in response to the same relative dose of bendroflumethiazide (Chen et al., 1994). Females also have a much larger reduction in simulated GFR than males $(-7.15 \% \pm$ $1.55 \%[\mathrm{M}]$ versus $-23.0 \% \pm 1.1 \%[\mathrm{~F}]$ ), which can be attributed to the drop in MAP.

\section{Overall response to different classes of antihypertensive drugs}

For all drugs, at a sufficiently high inhibition level, females have on average a greater relative decrease in simulated MAP than males, despite having a lower pre-drug MAP (Figures 3A, 3B, 4A, and 5A). In order of increasing benefit when compared with virtual males, virtual females respond better to $A C E i, A R B, C C B$, and TZD. This is due to the greater cardioprotective physiology of females (load shift in fluid and sodium transport from the proximal to the distal and collecting segments in the nephron; less excitatory RSNA [Ahmed and Layton, 2020]). Likewise, for all drugs, females require a lower simulated target inhibition level to reach an average MAP of $120 \mathrm{mmHg}$. This is due to both females having a lower pre-drug MAP and the cardioprotective physiology of females. To reach an average MAP of $120 \mathrm{mmHg}$, males and females require an inhibition level of $93 \%$ and $86 \%$ of $A C E i, 84 \%$ and $77 \%$ of ARB, and $88 \%$ and $77 \%$ of CCB, respectively. For TZD, females require an inhibition level of $40 \%$, whereas males are not able reach this MAP level. For the given theoretical range of inhibition level, TZD, ACEi, $A R B$, and $C C B$ is on average the increasing order of potential MAP reduction in males, whereas ACEi, TZD, ARB, and CCB is the one in females. ARB has a greater range of effectiveness than ACEi because it blocks the binding of Ang $I$ to its type 1 receptor directly, whereas with ACEi, Ang I can still be converted into Ang II through chymase (see Equation S66). This benefit of ARB over ACEi is even greater in females due to the vasodilatory effect of AT2R-bound Ang II, as explained above.

\section{Underlying pathophysiology is a highly accurate predictor of optimal choice of drug class}

To compare the different classes of antihypertensive drugs, we choose a simulated inhibition level that results in an average decrease in MAP by $20 \%$ across the virtual population as a normalization. We then construct a decision tree that predicts which drug causes the greatest decrease in simulated MAP based on one's pathophysiological variables (see transparent methods for full details). Responses to ACEi and ARB are similar. In both sexes, results for ARB can be applied to ACEi. At maximum inhibition, TZD is unable to produce a $20 \%$ reduction in MAP in males. Thus, the male virtual population is classified for ARB and CCB only, whereas the female virtual population is classified for ARB, CCB, and TZD only. The resulting decision trees for males and females are plotted in Figure 6. Of the eight pathophysiological variables, three are predicted to be sufficient for determining optimal treatment. For males, these variables are the myogenic effect, antidiuretic hormone concentration, and afferent arteriolar resistance (Figure 6A), whereas for females, these variables are the aldosterone concentration, plasma renin concentration, and myogenic effect (Figure 6B). The receiver operating characteristic (ROC) curves for ARB, CCB, and TZD in males and females are plotted in Figure 7. The area under the curve for males and females and for all drugs ranges from $88 \%-$ $96 \%$, indicating high accuracy of the classification model.

\section{DISCUSSION}

\section{Mechanistic model of spontaneously hypertensive rats}

We have developed the first sex-specific QSP model of primary hypertension in rats. The model is based upon previously published models of blood pressure regulation (Ahmed and Layton, 2020; Guyton et al., 1972; Hallow et al., 2014; Karaaslan et al., 2005; Leete and Layton, 2019), and the novelty herein lies in the formulation of the primary hypertensive model. Previous quantitative models have been developed for either a specific etiology of hypertension, such as renovascular, salt sensitive, impaired kidney function, etc. (Beard and Mescam, 2012; Clemmer et al, 2016, 2018; Hallow and Gebremichael, 2017b; Hallow et al., 2014; Leete and Layton, 2019), or by perturbing all parameters and classifying the resulting virtual population (Moss et al., 2012). Chen et al. developed sex-specific quantitative models of SHR kidneys (Chen et al., 2017). As primary hypertension accounts for $90 \%-95 \%$ of all human hypertension cases, it is important to have a quantitative model for this situation to be able to understand mechanisms and 
A

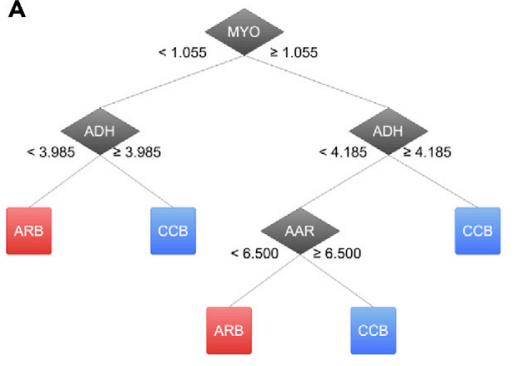

B

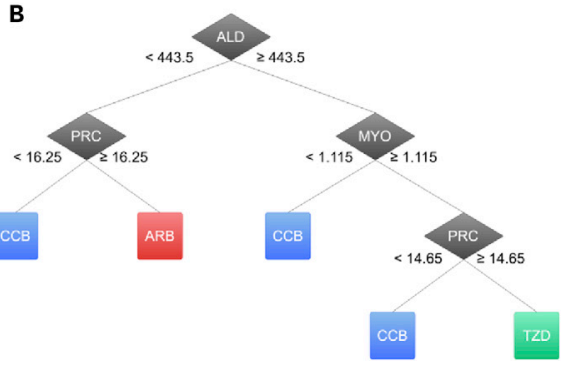

Figure 6. Decision trees for predicting optimal drug classes

(A) Decision tree in males. (B) Decision tree in females. MYO, myogenic effect (unitless); ADH, antidiuretic hormone concentration ( $\mu$ units $/ \mathrm{mL}) ; A A R$, afferent arteriolar resistance $(\mathrm{mmHg} /(\mathrm{mL} / \mathrm{min})) ; A L D$, aldosterone concentration (ng/L); PRC, plasma renin concentration ( $\mathrm{fmol} / \mathrm{mL}$ ); angiotensin receptor blocker (ARB); CCB, calcium channel blocker; TZD, thiazide diuretic. See also Figure S1.

guide treatment. Human models may provide direct clinical applications, whereas rat models are useful for aiding in the interpretation of rodent experiments. Thus, as a proof of concept, we developed this model for the SHR, which is the most commonly used animal model for human primary hypertension. Moreover, the more invasive nature of rodent experiments has allowed us to rigorously calibrate the model by distinguishing which variables and parameters to change and which to keep constant in relation to the pathophysiology of hypertension. We then used the resulting mechanistic model integrated with a machine learning model to study the differential effects of an individual's sex and pathophysiology on the response to treatment by administering different classes of antihypertensive drugs to the male and female virtual populations.

\section{Impact of sex on response to treatment}

Previously, we have utilized a quantitative model for blood pressure regulation to show how sexually dimorphic experimental results can be explained by sex-specific physiological mechanisms (Ahmed and Layton, 2020). The sex differences in blood pressure regulation in our previous work were calibrated from experimental data and based upon the current understanding among the physiologist community. They include (1) vasodilation of the renal afferent and efferent arterioles by AT2R-bound ANG II in the RAS (which is assumed to occur only in females), (2) sex differences in the RAS, (3) a less excitable and more easily repressed RSNA in females than in males, and (4) a load shift in females in renal sodium and fluid handling from the proximal to downstream segments of the nephron (Ahmed and Layton, 2020). This sex-specific normotensive model is the base from which we now develop the hypertensive model. There are some similarities in the distributions of the pathophysiological parameters in males and females (Figure 2). Interestingly, although we use the same methodology calibrated with experimental data in males and females (see transparent methods and Figure S2), we also observe some differences in the resulting distributions (Figure 2). Thus, differences between males and females in their hypertensive pathophysiological state is a consequence of the underlying sexually dimorphic mechanisms. As a result of accounting for sex differences in both blood pressure regulation mechanisms and hypertension pathophysiologies, we then observe sex differences in the severity of hypertension (Table 1) and in response to antihypertensive drugs (Figures 3A, 3B, 4A, and 5A).

Females have on average a greater relative decrease in MAP than males at sufficiently high inhibition levels for all drug classes (Figures 3A, 3B, 4A, and 5A). And this is despite having a lower pre-drug MAP. A greater response to drugs in females than in males is consistent with experimental results of administration of $A C E i$ (Dalpiaz et al., 2013; Gong et al., 1994), ARB (Rodrigues et al., 2006; Zimmerman et al., 2014), and TZD (Chen et al., 1994) in SHRs. (We could not find experimental data for administering CCB to female SHRs.) Model simulations indicate that this is due to their cardioprotective physiology, particularly, the most advantageous mechanisms in females being their less excitable RSNA and a load shift of sodium reabsorption from the proximal to distal and collecting segments in the nephron. We have a quantitative model that (1) incorporates sex-specific mechanisms in blood pressure regulation, which leads to (2) a sex-specific pathophysiology of hypertension, and as a result, (3) a sexually dimorphic response to antihypertensive drugs. Therefore, model results suggest that sex is a significant determinant of response to drugs and tailoring antihypertensive therapy to one's sex is more effective in lowering MAP in SHRs. 
A
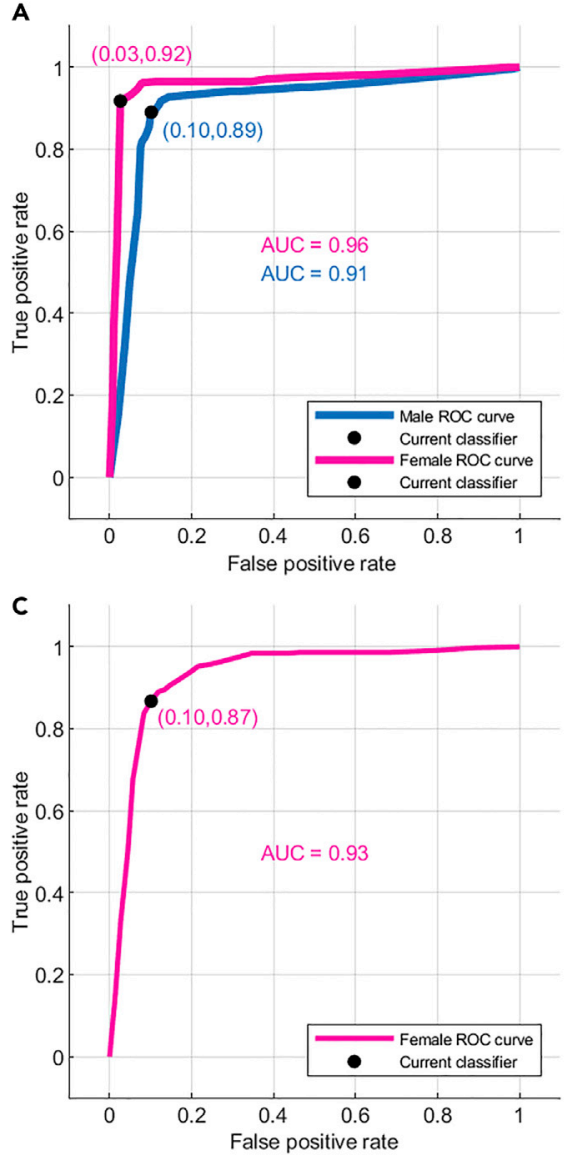

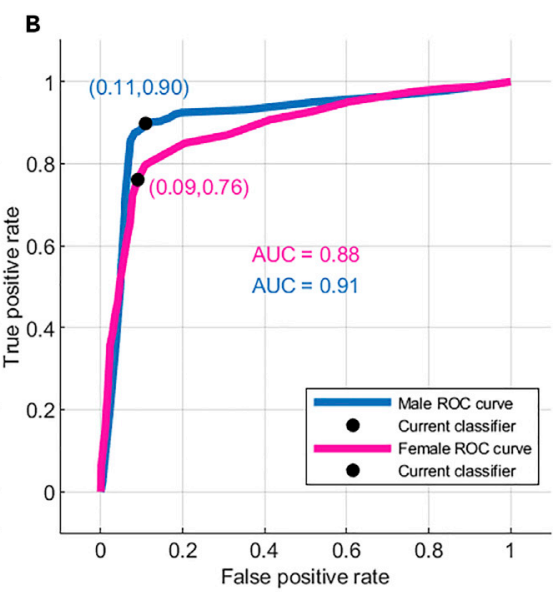

Figure 7. Receiver operating characteristic (ROC) curves

(A) ROC curves for males and females for choosing angiotensin receptor blocker (ARB). (B) ROC curves for males and females for choosing calcium channel blocker (CCB). (C) ROC curve for females for choosing thiazide diuretic (TZD). The current classifier is the calculated true- and false-positive rates for the decision tree in Figure 6. Area under the curve (AUC).

\section{Impact of pathophysiological profile on response to treatment}

Different underlying physiological mechanisms can have the same apparent physiological manifestation. Yet for different parameterizations of a model with similar variable values, there will be variation in responses to when the system is perturbed. This is particularly true for primary hypertension, which results from an aberrance in several regulators of blood pressure. Adopting the methodology from Barish et al. (2017), we create male and female virtual populations. All virtual individuals have key variable outputs within the physiological range, yet they have a wide spread in the baseline values of their pathophysiological parameters (Figure 2). Hence the virtual population has captured, in part, the genetic diversity in hypertension among SHRs.

Differences in underlying hypertension pathophysiologies can, in part, explain the variation in responses to different classes of antihypertensive drugs. Model simulations demonstrate that (1) incorporating variation in the pathophysiology of hypertension leads to a variation in MAP response to antihypertensive therapies, which in turn (2) causes a differential response to different classes of drugs for each individual, from which we can (3) classify which drug works best for different individuals based upon their pathophysiological profile. Points (1) and (2) have been explored by previous modeling studies in the context of secondary hypertension (Clemmer et al., 2018; Hallow and Gebremichael, 2017b; Hallow et al., 2014). Of the eight variables associated with the pathophysiology of hypertension, three are sufficient (myogenic response, antidiuretic hormone concentration, and afferent arteriolar resistance in males; aldosterone concentration, plasma renin concentration, and myogenic response in females) for predicting the optimal drug class with high 
accuracy (Figures 6 and 7). Therefore, model results suggest that one's pathophysiological profile provides a stronger indicator of which drug class can more effectively lower MAP in SHRs than choosing one at random. Additionally, predictor variables for optimal drug classes are different for males and females, providing another case for the impact of sex on MAP response to drugs.

\section{Clinical implications: personalized medicine}

Interpatient variability may be a contributing factor as to why success of treatment of hypertension is so low because the average recommendations may not be suitable for individual patients. Precision medicine aims to mitigate this problem by providing "the right patient with the right treatment at the right dose" (Currie and Delles, 2018). Quantitative techniques serve as useful tools in this effort as patient variability can be incorporated into model components. Initially, sufficient data from diverse patients is needed to calibrate quantitative models, but the added efficiency is in the predictive power in that these models can then be used to explore heterogeneity in response to various treatment protocols (Gibson et al., 2018). Using a QSP model of blood pressure regulation and hypertension, we show that sex and pathophysiological profile have significant impacts on the blood pressure-lowering capacity of different classes of antihypertensive drugs in SHRs.

Sex differences are observed in the mechanisms involved in blood pressure regulation and in the pathophysiologies causing hypertension in both humans and animals, including the SHR. As a result, the severity of hypertension and response to antihypertensive drugs differs among males and females (Kalibala et al., 2020; Reckelhoff, 2018). We have shown a sexually dimorphic response to different classes of antihypertensive drugs based upon sex-specific mechanisms in the SHR. This difference occurs when implicitly accounting for drug administration through inhibition of the target. The difference may be exacerbated, though, when explicitly accounting for administration of the drug because certain antihypertensive drugs have been shown to have different pharmacokinetics and pharmacodynamics (PKPD) in men and women (Kalibala et al., 2020). Accounting for drug administration explicitly through a PKPD model can then provide better guidelines for sex-specific dosing.

Overall, clinical data on sex differences in response to antihypertensive drugs is scarce because either women are underrepresented in these studies or sex-specific analyses were not performed (Kalibala et al., 2020). However, some observations can be noted. Men are more often prescribed ACEi and ARB, whereas women are more often prescribed CCB and TZD (Kalibala et al., 2020). This implicit preference could be due to certain classes of drugs having either less adverse effects or greater reduction in blood pressure in either sex. Women respond better than men to ACEi, ARB/TZD dual therapy, and CCB (Graziamaria et al., 2017; Kalibala et al., 2020), which is also reflected in our model results in SHRs. Women also have better cardiovascular outcomes than men when taking ARB (Graziamaria et al., 2017). Sexual dimorphism in response to antihypertensive drugs exists not only in terms of reduction of blood pressure but also in terms of adverse effects, which can be seen in women having more frequent adverse reactions to taking ACEi and TZD (Seeland and Regitz-Zagrosek, 2013). Adverse drug reactions are the main reason for interruption of therapy. This indicates an even greater need for considering sex when prescribing antihypertensive drugs. For better insights for sex differences in humans, one would need a human sex-specific quantitative model (Leete and Layton, 2019), although data for calibrating the model are difficult to obtain as opposed to with animal models.

Our model is a simplified model of blood pressure regulation that accounts for just the essential mechanisms. The goal of simulating drugs across the virtual population is thus to gain insight on whether there are correlations between pathophysiological profiles and response to treatment. Model simulations suggest that pathophysiological profiles in SHRs provide strong predictors for success of therapy, making this a potential area for precision medicine in the treatment of hypertension. The goal of this study is not to have a mapping between virtual and real individuals. For better insights in humans, one would need a quantitative model for human primary hypertension, although there are limitations in being able to apply as rigorous a formulation for human primary hypertension as we have for the SHR due to obvious restrictions in data. Future work is hence needed to better characterize primary hypertension in humans. Moreover, for a stronger mechanistic mapping between virtual and real individuals, a holistic model of human physiology would be needed. Continuing efforts are being made to create virtual populations in more comprehensive mechanistic quantitative models of human physiology, where the ultimate goal is to be able to have a stronger correspondence between virtual and real individuals (Clemmer et al., 2018; Hester 
et al., 2011; Moss et al., 2012). Such models may eventually be able to map a set of model parameters to a patient's sex, age, ethnicity, or pathophysiological profile and thus identify clinically relevant biomarkers for response to antihypertensive therapy.

\section{Limitations of the study}

In this study, we account for genetic diversity through differences in the pathophysiological mechanisms in hypertension. In other words, we account for heterogeneity in disease, but not population (or experimental cohort). To account for greater genetic diversity, differences in physiology should also be considered. One can try a hybrid approach by combining the methodologies in Clemmer et al. (2018) and Moss et al. (2012) with the one presented here. More specifically, first perturb all parameters as in Clemmer et al. (2018) and Moss et al. (2012), but impose stricter selection criteria for keeping a set of parameters, as done in this study. This will result in a normotensive virtual population. Then induce hypertension in this normotensive virtual population as done in this study. As we have only accounted for heterogeneity in disease, we were able to find correlations between pathophysiological profiles and response to different classes of antihypertensive drugs. The advantage of accounting for heterogeneity in population as well would be the ability to identify other physiological indicators of response to therapy. Moreover, several of the pathophysiological quantities that we have identified as accurate predictors of response to different classes of drugs are not readily measurable (Figure 6). Thus we present our analysis herein as a proof of concept of the impact of pathophysiological profile on the response to therapy. And adaptation for clinical relevance will require study of clinically relevant biomarkers (Asano, 2018).

We simulate the administration of drugs implicitly with target inhibition. Future work is to explicitly simulate the administration of drugs by coupling our QSP model with a detailed PKPD model for each of the drug classes considered (Clegg and Mac Gabhann, 2015; Gebremichael et al., 2019). The utility of this would be to predict change in blood pressure in response to the actual drug doses. Also, as aforementioned, sex differences exist in the PKPD of certain antihypertensive drugs (Kalibala et al., 2020). So, such a detailed model would also allow for a more accurate evaluation of sexual dimorphism in response to drugs. Future work also includes considering dual and triple therapies. Multiple therapies are commonly prescribed to hypertensive patients after the inability of monotherapy to get blood pressure under control (James et al., 2014). As disruption in several mechanisms contribute to the development of primary hypertension, multiple drug classes are needed for corrective action across the different regulators of blood pressure.

\section{Resource availability}

Lead contact

Further information and requests for resources should be directed to and will be fulfilled by the lead contact, Sameed Ahmed (sameed.ahmed@uwaterloo.ca).

Material availability

This study did not generate nor use any new or unique reagents.

Data and code availability

The code generated during this study are available on GitHub (https://github.com/sameed-ahmed/OSPMod-SHR.git).

\section{METHODS}

All methods can be found in the accompanying transparent methods supplemental file.

\section{SUPPLEMENTAL INFORMATION}

Supplemental information can be found online at https://doi.org/10.1016/j.isci.2021.102341.

\section{ACKNOWLEDGMENTS}

This research was supported by the Canada 150 Research Chair program and by the NSERC Discovery award to A.T.L. 


\section{AUTHOR CONTRIBUTIONS}

S.A. conceived and designed the research. S.A. and J.C.S. searched the literature. S.A. performed the simulations. S.A., J.C.S., and A.T.L. analyzed the simulated output. S.A., J.C.S., and A.T.L. interpreted the results of the simulations. S.A. prepared the figures. S.A. drafted the manuscript. S.A., J.C.S., and A.T.L. edited and revised manuscript. S.A., J.C.S., and A.T.L. approved final version of manuscript.

\section{DECLARATION OF INTERESTS}

The authors declare no competing interests.

Received: November 2, 2020

Revised: February 22, 2021

Accepted: March 17, 2021

Published: April 23, 2021

\section{REFERENCES}

Ahmed, S., Hu, R., Leete, J., and Layton, A.T (2019). Understanding sex differences in longterm blood pressure regulation: insights from experimental studies and computational modeling. Am. J. Physiol. Heart Circ. Physiol. 316, H1113-H1123.

Ahmed, S., and Layton, A.T. (2020). Sex-specific computational models for blood pressure regulation in the rat. Am. J. Physiol. Renal Physiol. 318, F888-F900, https://doi.org/10.1152/ ajprenal.00376.2019.

Antonaccio, M.J., Rubin, B., and Horovitz, Z.P. (1980). Effects of captopril in animal models of hypertension. Clin. Exp. Hypertens. 2, 613-637, https://doi.org/10.3109/10641968009037133.

Antonaccio, M.J., Rubin, B., Horovitz, Z.P., Laffan, R.J., Goldberg, M.E., High, J.P., Harris, D.N., and Zaidi, I. (1979). Effects of chronic treatment with captopril (SO 14, 225), an orally active inhibitor of angiotensin I-converting enzyme, in spontaneously hypertensive rats. J. Pharmacol. 29, 285-294, https://doi.org/10.1254/jjp.29.285.

Arendshorst, W.J., and Beierwaltes, W.H. (1979). Renal and nephron hemodynamics in spontaneously hypertensive rats. Am. J. Physiology-Renal Physiol. 236, F246-F251, https://doi.org/10.1152/ajprenal.1979.236.3. F246.

Asano, Y. (2018). How to eliminate uncertainty in clinical medicine - clues from creation of mathematical models followed by scientific data mining. EBioMedicine 34, 12-13, https://doi.org/ 10.1016/j.ebiom.2018.07.001.

Badyal, D.K., Dadhich, A.P., and Lata, H. (2003) Animal models of hypertension and effect of drugs. Indian J. Pharmacol. 35, 349.

Barish, S., Ochs, M.F., Sontag, E.D., and Gevertz, J.L. (2017). Evaluating optimal therapy robustness by virtual expansion of a sample population, with a case study in cancer immunotherapy. Proc. Natl. Acad. Sci. U S A 114, E6277-E6286, https://doi. org/10.1073/pnas.1703355114.

Beard, D.A., and Mescam, M. (2012). Mechanisms of pressure-diuresis and pressure-natriuresis in Dahl salt-resistant and Dahl salt-sensitive rats. BMC Physiol. 12, 6, https://doi.org/10.1186/14726793-12-6.
Beierwaltes, W.H., Arendshorst, W.J., and Klemmer, P.J. (1982). Electrolyte and water balance in young spontaneously hypertensive rats. Hypertension 4, 908-915, https://doi.org/10 1161/01.HYP.4.6.908.

Brinson, K.N., Rafikova, O., and Sullivan, J.C. (2014). Female sex hormones protect against saltsensitive hypertension but not essential hypertension. Am. J. Physiol. Regul. Integr. Comp. Physiol. 307, R149-R157, https://doi.org/ 10.1152/ajpregu.00061.2014.

Bunkenburg, B., Schnell, C., Baum, H.P., Cumin, F., and Wood, J.M. (1991). Prolonged angiotensin Il antagonism in spontaneously hypertensive rats. Hemodynamic and biochemical consequences. Hypertension 18, 278-288, https://doi.org/10. 1161/01.HYP.18.3.278.

Chen, Y., Sullivan, J.C., Edwards, A., and Layton, A.T. (2017). Sex-specific computational models of the spontaneously hypertensive rat kidneys: factors affecting nitric oxide bioavailability. Am. J Physiol. Renal Physiol. 313, F174-F183, https:// doi.org/10.1152/ajprenal.00482.2016.

Chen, Z., Vaughn, D.A., and Fanestil, D.D. (1994) Influence of gender on renal thiazide diuretic receptor density and response. JASN 5, 11121119

Clegg, L.E., and Mac Gabhann, F. (2015) Molecular mechanism matters: benefits of mechanistic computational models for drug development. Pharmacol. Res. 99, 149-154, https://doi.org/10.1016/j.phrs.2015.06.002.

Clemmer, J.S., Hester, R.L., and Pruett, W.A (2018). Simulating a virtual population's sensitivity to salt and uninephrectomy. Interf. Focus 8 , 20160134, https://doi.org/10.1098/rsfs.2016. 0134

Clemmer, J.S., Pruett, W.A., Coleman, T.G., Hall, J.E., and Hester, R.L. (2016). Mechanisms of blood pressure salt sensitivity: new insights from mathematical modeling. Am. J. Physiol. Regul. Integr. Comp. Physiol. 312, R451-R466, https:// doi.org/10.1152/ajpregu.00353.2016.

Currie, G., and Delles, C. (2018). Precision medicine and personalized medicine in cardiovascular disease. In Sex-Specific Analysis of Cardiovascular Function, Advances in Experimental Medicine and Biology, P.L.M.
Kerkhof and V.M. Miller, eds. (Springer International Publishing), pp. 589-605, https:// doi.org/10.1007/978-3-319-77932-4_36.

Dalpiaz, P.L.M., Lamas, A.Z., Caliman, I.F., Medeiros, A.R.S., Abreu, G.R., Moysés, M.R., Andrade, T.U., Alves, M.F., Carmona, A.K., and Bissoli, N.S. (2013). The chronic blockade of angiotensin I-converting enzyme eliminates the sex differences of serum cytokine levels of spontaneously hypertensive rats. Braz. J. Med. Biol. Res. 46, 171-177, https://doi.org/10.1590/ $1414-431 \times 20122472$

Fenoy, F.]., Milicic, I., Smith, R.D., Wong, P.C., Timmermans, P.B.M.W.M., and Roman, R. (1991). Effects of DuP 753 on renal function of normotensive and spontaneously hypertensive rats. Am. J. Hypertens. 4, 321S-326S, https://dol. org/10.1093/ajh/4.4.321S.

Francischetti, A., Ono, H., and Frohlich, E.D (1998). Renoprotective effects of felodipine and/ or enalapril in spontaneously hypertensive rats with and without L-NAME. Hypertension 31, 795-801, https://doi.org/10.1161/01.HYP.31.3 795.

Freeman, R.H., Davis, J.O., Aharon, N.V., Ulick, S., and Weinberger, M.H. (1975). Control of aldosterone secretion in the spontaneously hypertensive rat. Circ. Res. 37, 66-71, https://doi. org/10.1161/01.RES.37.1.66.

Gebremichael, Y., Lahu, G., Vakilynejad, M., and Hallow, K.M. (2019). Benchmarking renin suppression and blood pressure reduction of direct renin inhibitor imarikiren through quantitative systems pharmacology modeling J. Pharmacokinet. Pharmacodyn. 46, 15-25, https://doi.org/10.1007/s10928-018-9612-y.

Gibson, E., Bretz, F., Looby, M., and Bornkamp, B. (2018). Key aspects of modern, quantitative drug development. Stat. Biosci. 10, 283-296, https:// doi.org/10.1007/s12561-017-9203-2.

Gong, G., Dobin, A., McArdle, S., Sun, L., Johnson, M.L., and Pettinger, W.A. (1994). Sex influence on renal alpha 2-adrenergic receptor density in the spontaneously hypertensive rat. Hypertension 23, 607-612, https://doi.org/10 1161/01.hyp.23.5.607.

Graziamaria, C., Immacolata, A., Klara, K., Michele, C., Anna, L., and Nicola, F. (2017). 
Gender differences in response to therapy for cardiovascular diseases. Curr.

Pharmacogenomics Person. Med. 15, https://doi. org/10.2174/1875692115666170508153322.

Guyton, A.C., Coleman, T.G., and Granger, H.J. (1972). Circulation: overall regulation. Annu. Rev. Physiol. 34, 13-44, https://doi.org/10.1146/ annurev.ph.34.030172.000305.

Hallow, K., and Gebremichael, Y. (2017a). A quantitative systems physiology model of renal function and blood pressure regulation: model description: a quantitative systems physiology model. CPT: Pharmacometrics Syst. Pharmacol. 6 , 383-392, https://doi.org/10.1002/psp4.12178.

Hallow, K., and Gebremichael, Y. (2017b). A quantitative systems physiology model of renal function and blood pressure regulation: application in salt-sensitive hypertension: a quantitative systems physiology model. CPT: Pharmacometrics Syst. Pharmacol. 6, 393-400, https://doi.org/10.1002/psp4.12177.

Hallow, K.M., Lo, A., Beh, J., Rodrigo, M., Ermakov, S., Friedman, S., de Leon, H., Sarkar, A., Xiong, Y., Sarangapani, R., et al. (2014). A modelbased approach to investigating the pathophysiological mechanisms of hypertension and response to antihypertensive therapies: extending the Guyton model. Am. J. Physiol. Regul. Integr. Comp. Physiol. 306, R647-R662, https://doi.org/10.1152/ajpregu.00039.2013.

Hester, R.L., Brown, A.J., Husband, L., Iliescu, R., Pruett, D., Summers, R., and Coleman, T.G. (2011). HumMod: a modeling environment for the simulation of integrative human physiology. Front. Physiol. 2, https://doi.org/10.3389/fphys 2011.00012.

Hu, R., McDonough, A.A., and Layton, A.T. (2020). Sex differences in solute transport along the nephrons: effects of $\mathrm{Na}+$ transport inhibition. Am. J. Physiol. Renal Physiol. 319, F487-F505, https://doi.org/10.1152/ajprenal.00240.2020.

Hu, R., McDonough, A.A., and Layton, A.T. (2019). Functional implications of the differences in transporters' abundance along the rat nephron: modeling and analysis. Am. J. Physiol. Renal Physiol. https://doi.org/10.1152/ajprenal.00352. 2019

James, P.A., Oparil, S., Carter, B.L., Cushman W.C., Dennison-Himmelfarb, C., Handler, J., Lackland, D.T., LeFevre, M.L., MacKenzie, T.D. Ogedegbe, O., et al. (2014). 2014 evidencebased guideline for the management of high blood pressure in adults: report from the panel members appointed to the eighth Joint national committee (JNC 8). JAMA 311, 507-520, https:// doi.org/10.1001/jama.2013.284427.

Johns, E.J. (1988). A study of the renal actions of amlodipine in the normotensive and spontaneously hypertensive rat. Br. J. Pharmacol. 94, 311-318, https://doi.org/10.1111/j.1476-5381. 1988.tb11532.x

Kalibala, J., Pechère-Bertschi, A , and Desmeules, J. (2020). Gender differences in cardiovascular pharmacotherapy-the example of hypertension: a mini review. Front. Pharmacol. 11, https://doi. org/10.3389/fphar.2020.00564.

Karaaslan, F., Denizhan, Y., Kayserilioglu, A., and Gulcur, H.O. (2005). Long-Term mathematical model involving renal sympathetic nerve activity, arterial pressure, and sodium excretion. Ann. Biomed. Eng. 33, 1607-1630, https://doi.org/10. 1007/s10439-005-5976-4.

Kohara, K., Mikami, H., Okuda, N., and Ogihara, T. (1993). Peripheral vs. Central blockade of the renin- angiotensin system in spontaneously hypertensive rats: comparison of novel AT receptor antagonist TCV-116 with angiotensin converting enzyme inhibitor delapril. Hypertens. Res. 16, 239-246, https://doi.org/10.1291/hypres. 16.239

Komatsu, K., Numabe, A., Ono, Y., and Frohlich, E.D. (1996). Hydrochlorothiazide increases efferent glomerular arteriolar resistance in spontaneously hypertensive rats. J. Cardiovasc. Pharmacol. Ther. 1, 57-64, https://doi.org/10. $1177 / 107424849600100109$

Lai, F.M., Tanikella, T., Herzlinger, H., Goldstein, B., Chan, P.S., and Cervoni, P. (1982). Studies on the mechanism of the enhancement of the antihypertensive activity of captopril by A diuretic in spontaneously hypertensive rats. Clin. Exp. Hypertens. A 4, 1001-1018, https://doi.org/10 3109/10641968209060768.

Layton, A.T., and Sullivan, J.C. (2018). Recent advances in sex differences in kidney function. Am. J. Physiol. Renal Physiol. 316, F328-F331. https://doi.org/10.1152/ajprenal.00584.2018.

Leete, J., Gurley, S., and Layton, A.T. (2018). Modeling sex differences in the renin angiotensin system and the efficacy of antihypertensive therapies. Comput. Chem. Eng. 112, 253-264, https://doi.org/10.1016/j.compchemeng.2018. 02.009

Leete, J., and Layton, A.T. (2019). Sex-specific long-term blood pressure regulation: modeling and analysis. Comput. Biol. Med. 104, 139-148, https://doi.org/10.1016/j.compbiomed.2018.11. 002

Li, Q., McDonough, A.A., Layton, H.E., and Layton, A.T. (2018). Functional implications of sexual dimorphism of transporter patterns along the rat proximal tubule: modeling and analysis. Am. J. Physiol. Renal Physiol. 315, F692-F700, https://doi.org/10.1152/ajprenal.00171.2018.

Messing, M., Van Essen, H., Smith, T.L., Smits, J.F.M., and Struyker-Boudier, H.A.J. (1991). Microvascular actions of calcium channel antagonists. Eur. J. Pharmacol. 198, 189-195 https://doi.org/10.1016/0014-2999(91)90620-6.

Mizuno, K., Niimura, S., Tani, M., Haga, H. Gomibuchi, T Sanada, H, and Fukuchi, S (1992a). Antihypertensive and hormonal activity of MK 954 in spontaneously hypertensive rats. Eur. J. Pharmacol. 215, 305-308, https://doi.org/ 10.1016/0014-2999(92)90045-6.

Mizuno, K., Tani, M., Hashimoto, S., Niimura, S., Sanada, H., Watanabe, H., Ohtsuki, M., and Fukuchi, S. (1992b). Effects of losartan, a nonpeptide angiotensin II receptor antagonist, on cardiac hypertrophy and the tissue angiotensin II content in spontaneously hypertensive rats. Life Sci. 51, 367-374, https:// doi.org/10.1016/0024-3205(92)90589-H.

Moss, R., Grosse, T., Marchant, I., Lassau, N. Gueyffier, F., and Thomas, S.R. (2012). Virtual patients and sensitivity analysis of the Guyton model of blood pressure regulation: towards individualized models of whole-body physiology. PLoS Comput. Biol. 8, e1002571, https://doi.org/ 10.1371/journal.pcbi.1002571.

Mougenot, N., Mediani, O., and Lechat, P. (2005). Bisoprolol and hydrochlorothiazide effects on cardiovascular remodeling in spontaneously hypertensive rats. Pharmacol. Res. 51, 359-365, https://doi.org/10.1016/j.phrs.2004.10.010.

Oparil, S., Acelajado, M.C., Bakris, G.L., Berlowitz, D.R., Cífková, R., Dominiczak, A.F., Grassi, G. Jordan, J., Poulter, N.R., Rodgers, A., and Whelton, P.K. (2018). Hypertension. Nat. Rev. Dis. Primers 4, https://doi.org/10.1038/nrdp.2018.14.

Reckelhoff, J.F. (2018). Sex differences in regulation of blood pressure. In Sex-Specific Analysis of Cardiovascular Function, Advances in Experimental Medicine and Biology, P.L.M. Kerkhof and V.M. Miller, eds. (Springer International Publishing), pp. 139-151, https:// doi.org/10.1007/978-3-319-77932-4_9.

Richer, C., Doussau, M.-P., and Giudicelli, J.-F. (1987). Systemic and regional hemodynamic profile of five angiotensin I converting enzyme inhibitors in the spontaneously hypertensive rat. Am. J. Cardiol. 59, D12-D17, https://doi.org/10. 1016/0002-9149(87)90046-4.

Ritchey, M.D., Gillespie, C., Wozniak, G., Shay, C.M., Thompson-Paul, A.M., Loustalot, F., and Hong, Y. (2018). Potential need for expanded pharmacologic treatment and lifestyle modification services under the 2017 ACC/AHA Hypertension Guideline. J. Clin. Hypertens. 20, 1377-1391, https://doi.org/10.1111/jch.13364.

Rodrigues, S.F.de P., dos Santos, R.A., SilvaAntonialli, M.M., Scavone, C., Nigro, D. Carvalho, M.H.C., de Cássia Tostes, R., and Fortes, Z.B. (2006). Differential effect of losartan in female and male spontaneously hypertensive rats. Life Sci. 78, 2280-2285, https://doi.org/10. 1016/j.lfs.2005.09.049.

Seeland, U., and Regitz-Zagrosek, V. (2013). Sex and gender differences in cardiovascular drug therapy. In Sex and Gender Differences in Pharmacology, Handbook of Experimental Pharmacology, V. Regitz-Zagrosek, ed. (Springer Berlin Heidelberg), pp. 211-236, https://doi.org/ 10.1007/978-3-642-30726-3_11.

Smith, T.L., and Hutchins, P.M. (1979). Central hemodynamics in the developmental stage of spontaneous hypertension in the unanesthetized rat. Hypertension 1, 508-517, https://doi.org/10. 1161/01.HYP.1.5.508.

Sullivan, J.C., Bhatia, K., Yamamoto, T., and Elmarakby, A.A. (2010). Angiotensin (1-7) receptor antagonism equalizes angiotensin IIinduced hypertension in male and female spontaneously hypertensive rats. Hypertension 56, 658-666, https://doi.org/10.1161/ HYPERTENSIONAHA.110.153668.

Unger, T., Moursi, M., Ganten, D., Hermann, K and Lang, R.E. (1986). Antihypertensive action of the converting enzyme inhibitor perindopril (S9490-3) in spontaneously hypertensive rats: comparison with enalapril (MK421) and ramipril (HOE498). J. Cardiovasc. Pharmacol. 8, 276-285. 
Veiras, L.C., Girardi, A.C.C., Curry, J., Pei, L., Ralph, D.L., Tran, A., Castelo-Branco, R.C., Pastor-Soler, N., Arranz, C.T., Yu, A.S.L., and McDonough, A.A. (2017). Sexual dimorphic pattern of renal transporters and electrolyte homeostasis. JASN 28, 3504-3517, https://doi. org/10.1681/ASN.2017030295.

Virani Salim, S., Alonso, A., Benjamin Emelia, J. Bittencourt Marcio, S., Callaway Clifton, W.

Carson April, P., Chamberlain Alanna, M., Chang Alexander, R., Cheng, S., Delling Francesca, N., Djousse Luc, et al.. (2020). Heart disease and stroke statistics-2020 update: a report from the American heart association. Circulation 141, e139-e596, https://doi.org/10.1161/CIR. 0000000000000757
Wong, P.C., Price, W.A., Chiu, A.T., Duncia, J.V., Carini, D.J., Wexler, R.R., Johnson, A.L., and Timmermans, P.B.M.W.M. (1991). In vivo pharmacology of DuP 753. Am. J. Hypertens. 4, 288S-298S, https://doi.org/10.1093/ajh/4.4.288S.

Wyss, J.M., Chen, Y.F., Jin, H., Gist, R., and Oparil, S. (1987). Spontaneously hypertensive rats exhibit reduced hypothalamic noradrenergic input after $\mathrm{NaCl}$ loading. Hypertension 10, 313-320, https:/ doi.org/10.1161/01.HYP.10.3.313.

Wyss, J.M., Roysommuti, S., King, K., Kadisha, I., Regan, C.P., and Berecek, K.H. (1994). Saltinduced hypertension in normotensive spontaneously hypertensive rats. Hypertension
23, 791-796, https://doi.org/10.1161/01.HYP.23. 6.791.

Yonetani, Y., Iwaki, K., Ishii, M., and Harada, H. (1987). A new diuretic that does not reduce renal handling of uric acid in rats, S-8666. Jpn. J. Pharmacol. 43, 399-405, https://doi.org/10.1254/ j.jp.43.399.

Zimmerman, M.A., Harris, R.A., and Sullivan, J.C. (2014). Female spontaneously hypertensive rats are more dependent on ANG (1-7) to mediate effects of low-dose AT, receptor blockade than males. Am. J. Physiol. Renal Physiol. 306, F1136F1142, https://doi.org/10.1152/ajprenal.00677. 2013. 
iScience, Volume 24

Supplemental information

Impact of sex and pathophysiology

on optimal drug choice in hypertensive rats:

Quantitative insights for precision medicine

Sameed Ahmed, Jennifer C. Sullivan, and Anita T. Layton 


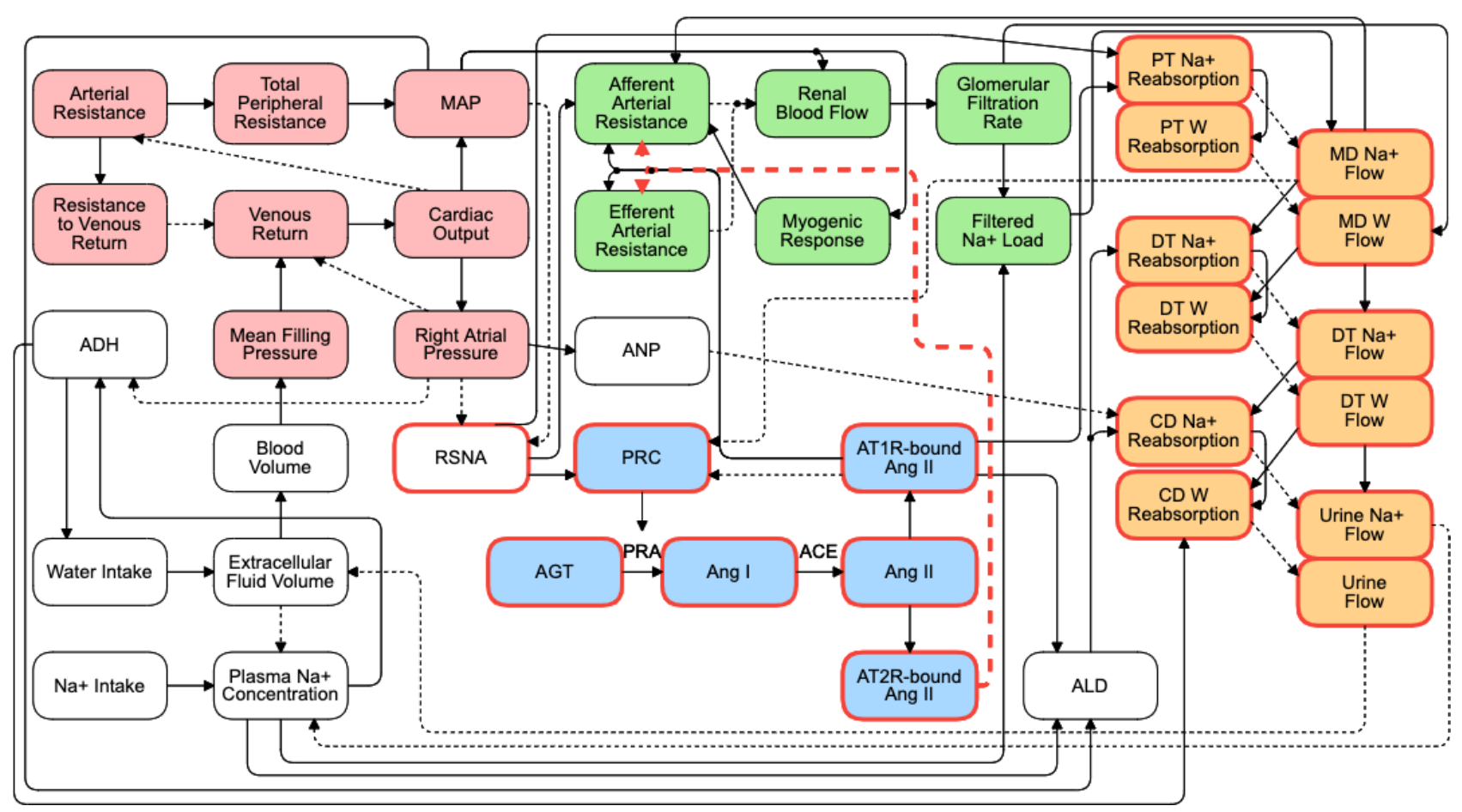

Figure S1: Schematic model of blood pressure regulation. Related to Figures 1-7. Pink nodes denote variables that describe cardiovascular function; green nodes, renal hemodynamics; orange nodes, renal $\mathrm{Na}+$ and fluid handling; blue nodes, renin angiotensin system. Red outlines denote sex-specific components. ADH, antidiuretic hormone; MAP, mean arterial pressure; ANP, atrial natriuretic peptide; RSNA, renal sympathetic nerve activity; PRC, plasma renin concentration; PRA, plasma renin activity; AGT, angiotensinogen; Ang I, angiotensin I; Ang II, angiotensin II; AT1R-bound Ang II, angiotensin II type 1 receptor bound angiotensin II; AT2R-bound Ang II, angiotensin II type 2 receptor bound angiotensin II; ALD, aldosterone. Reproduced with permission from Ref. (Ahmed and Layton 2020). 


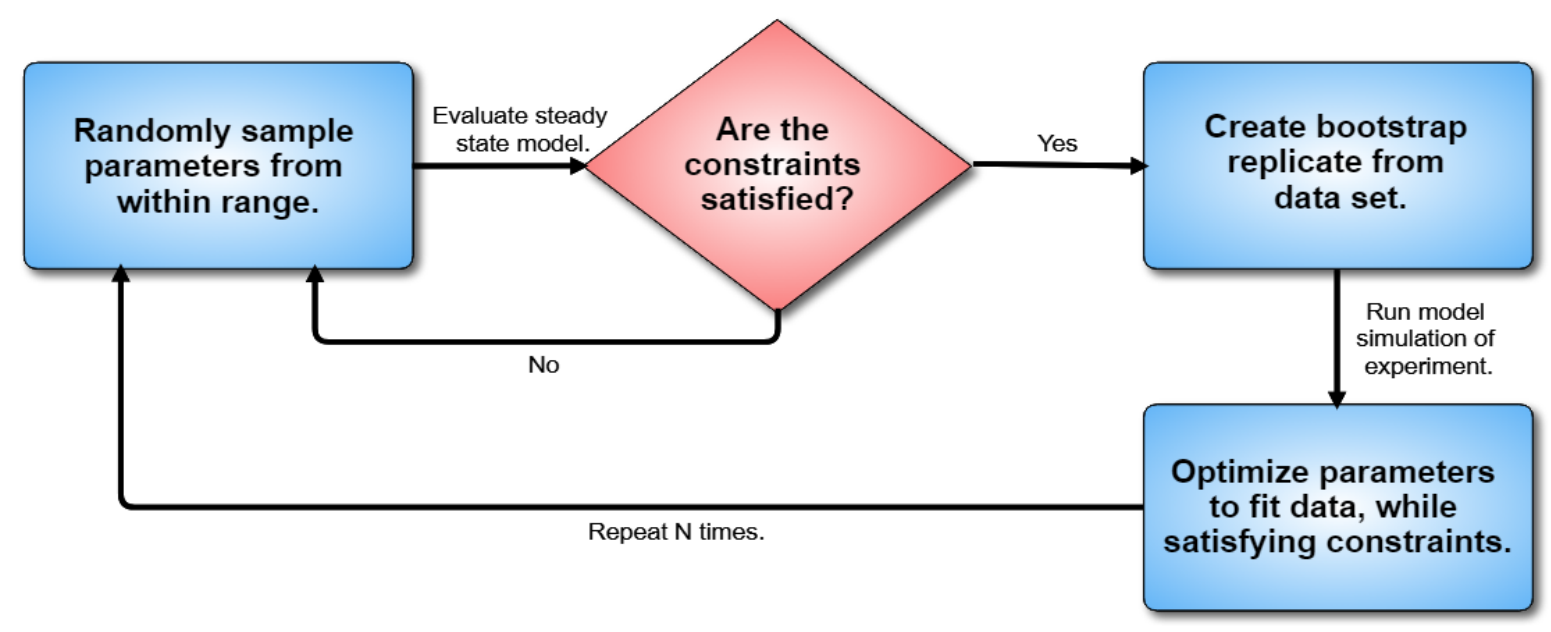

Figure S2: Flowchart of creating the spontaneously hypertensive rat (SHR) virtual population. Related to Figures 1 and 2. The constraints are that the model outputs for key physiological variables at baseline and after sodium loading are within experimental range. The data is the time course mean arterial pressure response to angiotensin II infusion. $\mathrm{N}$ is the size of the virtual population. 


\section{Transparent Methods}

\section{Methods}

\section{Sex-specific quantitative systems pharmacology model of blood pressure regulation in the rat}

The model presented here is based upon our previously published sex-specific computational model for blood pressure regulation in the rat (Ahmed and Layton 2020). The model is a large system of coupled nonlinear algebraic differential equations. It describes the interactions among the cardiovascular, renal, renal sympathetic nervous, and renin angiotensin systems, and how these systems regulate blood pressure and kidney function in response to various perturbations. A schematic diagram of the model is given in Figure S1. All model equations are given in the next section. And all parameter and variable values are given in Table S1.

\section{Creating the virtual spontaneously hypertensive rat}

The spontaneously hypertensive rat (SHR) is the most popular animal model of human primary hypertension. This strain was developed by breeding several generations of hypertensive Wistar-Kyoto rats (WKY) among an otherwise normotensive population. The offspring of the resulting strain then unanimously developed hypertension upon reaching a certain age (Badyal, Dadhich, and Lata 2003 Okamoto and Aoki 1963). Hence, this animal model is not hypertensive due to a specific pathology or environmental factor (e.g., increased sodium sensitivity, increased vascular resistance, impaired kidney function, etc.). Rather, it is genetically hypertensive. Several studies have physiologically characterized the SHR and probed the underlying pathologies responsible for the development of hypertension (W. J. Arendshorst and W. H. Beierwaltes 1979, W H Beierwaltes, W J Arendshorst, and Klemmer 1982 Bianchi et al. 1974, Freeman et al. 1975. Judy and Farrell 1979 Liard 1977, Peeters 2014). As it turns out, it is the combination of a multitude of pathophysiologies that collectively contribute to the development of hypertension in the SHR. Moreover, much data exists in comparing physiological measurements between the SHR and WKY. We parse the literature to collect this data and translate it into the model. The pathophysiologies inform perturbations of model parameters, and the physiological measurements inform constraints on model variables. Most of this data is from male rats. We therefore assume that similar differences exist between female SHR and WKY as they do between male SHR and WKY.

As compared to their normotensive controls WKYs, SHRs are characterized by an elevated mean arterial pressure (MAP) and similar cardiac output (Badyal, Dadhich, and Lata 2003). SHRs have similar body weight to WKYs, and thus similar blood volume (W H Beierwaltes, W J Arendshorst, and Klemmer 1982 Lee and Blaufox 1985). Assuming Poiseuille flow, MAP (denoted $P_{m a}$ ) is expressed in terms of the cardiac output $\left(\Phi_{c o}\right)$ and total peripheral vascular resistance $\left(R_{t p}\right)$,

$$
P_{m a}=\Phi_{c o} \times R_{t p}
$$

Therefore, SHRs have an elevated total peripheral vascular resistance in proportion to the elevated MAP. SHRs and WKYs have similar renal blood flow (RBF) (W. J. Arendshorst and W. H. Beierwaltes 1979). By the same reasoning, renal vascular resistance $\left(R_{r}\right)$ in SHRs is elevated in proportion to the elevation in MAP. Renal vascular resistance is expressed in terms of the afferent $\left(R_{A A}\right)$ and efferent $\left(R_{E A}\right)$ arteriolar resistances,

$$
R_{r}=R_{A A}+R_{E A}
$$

As compared to WKYs, SHRs have an elevated afferent arteriolar resistance, but similar efferent arteriolar resistance (W. J. Arendshorst and W. H. Beierwaltes 1979). The afferent arteriolar resistance is the product of its baseline value $\left(R_{A A}^{b l}\right)$, myogenic response $\left(\Sigma_{m y o}\right)$, and several autoregulatory mechanisms.

$$
R_{A A}=R_{A A}^{b l} \times \Sigma_{m y o} \times(\text { other terms }),
$$

The myogenic response is given in terms of the glomerular hydrostatic pressure $\left(P_{g h}\right)$ by

$$
\Sigma_{m y o}=a_{m y o}+\frac{b_{m y o}}{1+c_{m y o} e^{-d_{m y o}\left(P_{g h}-P_{g h}^{b l}\right)}}
$$


where $a_{m y o}$ is the minimal response, $b_{m y o}+a_{m y o}$ is the maximal response, $c_{m y o}$ is the horizontal shift, $d_{m y o}$ is the slope of the response, and $P_{g h}^{b l}$ is the baseline glomerular hydrostatic pressure. In isolated perfusion studies, the afferent arteriole in SHRs has been shown to constrict more than that of the WKYs in response to the same elevation in perfusion pressure (Ito, Juncos, and Carretero 1992). Therefore, SHRs have an overactive myogenic response.

To demonstrate that the kidney plays a key role in the regulation of blood pressure, transplantation studies have been conducted where the kidneys of SHRs and WKYs have been swapped. The donation of a normotensive kidney into a hypertensive recipient results in an attenuation, but not elimination, of hypertension in the recipient. Furthermore, the reverse transplantation results in hypertension in the recipient, but at an attenuated level from that of the donor (Bianchi et al. 1974). Thus, aberrant regulation of blood pressure by the kidney plays a key role in the development of hypertension in the SHR, but it is not the sole contributor. SHRs and WKYs have similar glomerular filtration rate (GFR) and urine output and sodium excretion (W. J. Arendshorst and W. H. Beierwaltes 1979). As a model assumption, we then assume that the renal transport parameters are the same. GFR $\left(\Phi_{g f i l t}\right)$ is expressed in terms of the perfusion pressure $\left(P_{f}\right)$ and glomerular capillary coefficient $\left(C_{g c f}\right)$,

$$
\Phi_{g f i l t}=P_{f} \times C_{g c f} .
$$

Perfusion pressure is similar in SHRs and WKYs (W. J. Arendshorst and W. H. Beierwaltes 1979). As a result, the glomerular capillary coefficient, which in part captures the material property of the glomerulus, is similar. Thus, it is reasonable to assume that SHRs have the same number of functioning nephrons as WKYs with no glomerular damage.

The sympathetic nervous system is another key regulator of blood pressure. In particular, renal sympathetic nerve activity (RSNA) acts in a multifaceted way: it modifies the resistance of the renal vasculature, renin secretion, and sodium reabsorption in the nephron. Compared to WKYs, SHRs have elevated RSNA, and renal denervation delays the onset of hypertension (Judy and Farrell 1979 Liard 1977). Aldosterone plays an important role in blood pressure regulation by regulating sodium reabsorption in the distal and collecting segments of the nephron. SHRs have elevated aldosterone secretion in comparison to WKYs (Freeman et al. 1975). Another major regulator of blood pressure is the renin angiotensin system (RAS), which acts by modifying the resistance of the renal vasculature, aldosterone secretion, and sodium reabsorption in the nephron. SHRs have greater plasma renin activity than WKYs (Peeters 2014). Antidiuretic hormone affects blood pressure regulation by adjust extracellular fluid levels through thirst and water reabsorption in the nephron; the level of antidiuretic hormone is higher in SHRs than in WKYs (Crofton et al. 1978). WKYs and SHRs have similar plasma sodium and potassium concentrations (Freeman et al. 1975).

Synthesizing these experimental findings in SHRs, we perturb select model parameters to create the SHR virtual population: those related to vascular resistance, strength of myogenic response, and baseline RSNA, aldosterone secretion rate, renin secretion rate, and antidiuretic hormone secretion rate. The decision to perturb these parameters while keep all others fixed is based upon experimental data characterizing the etiology of hypertension in the SHR, as mentioned above.

Ranges for parameters related to vascular resistance are determined from constraints on other physiological variables. For example, $\operatorname{RBF}\left(\Phi_{r b}\right)$ is related to $\operatorname{MAP}\left(P_{m a}\right)$ and renal vascular resistance $\left(R_{r}\right)$ by

$$
\Phi_{r b}=\frac{P_{m a}}{R_{r}},
$$

from which we express renal vascular resistance as

$$
R_{r}=\frac{P_{m a}}{\Phi_{r b}} .
$$

MAP in WKYs is about $100 \mathrm{mmHg}$ and can go up to about $150 \mathrm{mmHg}$ in SHRs in the age group we are modeling (Table S1). Meanwhile, RBF levels are similar. Let $R_{r}$ and $R_{r}^{\prime}$ denote the renal vascular resistance in WKY and SHR, respectively, with the same terminology applied to all other variables. From having physiological constraints on MAP and RBF, we then determine an upper bound on the range of renal vascular resistance as

$$
\begin{aligned}
R_{r}^{\prime} & =\frac{P_{m a}^{\prime}}{\Phi_{r b}^{\prime}} \\
& \leq \frac{150 / 100 \times P_{m a}}{\Phi_{r b}}
\end{aligned}
$$




$$
=1.5 \times R_{r} .
$$

As compared to WKY, afferent arteriolar resistance in elevated in SHR, while efferent arteriolar resistance is similar. Let $\alpha_{r}$ denote the factor by which afferent arteriolar resistance is elevated. Then from the preceding, we have

$$
\begin{aligned}
R_{r}^{\prime} & \leq 1.5 \times R_{r} \\
R_{A A}^{\prime}+R_{E A}^{\prime} & \leq 1.5 \times\left(R_{A A}+R_{E A}\right) \\
\alpha_{r} \times R_{A A}+R_{E A} & \leq 1.5 \times R_{A A}+1.5 \times R_{E A} .
\end{aligned}
$$

Rearranging to solve for $\alpha_{r}$ and plugging in the values (Table S1) yields

$$
\begin{aligned}
\alpha_{r} & \leq 0.5 \times \frac{R_{E A}}{R_{A A}}+1.5 \\
& =2.32 .
\end{aligned}
$$

To allow a safe range for this value, we set the upper bound for the sampling range of $R_{A A}$ to be up to three-fold $\left(\alpha_{r} \leq 3\right)$. We derive ranges for the other perturbed parameters related to vascular resistance through a similar analysis.

We determine the range for the strength of the myogenic response from experimental results in isolated microperfused afferent arterioles (Ito, Juncos, and Carretero 1992). Since the study was conducted in an isolated arteriole, we attribute the autoregulatory response to changes in perfusion pressure only to the myogenic response, and not to any other downstream mechanisms that would activate in intact circulation (such as RSNA or tubuloglomerular feedback). In this study, a greater contractile response to elevated perfusion pressure was observed in SHRs than in WKYs. At the largest perfusion pressure induced, the authors reported that the luminal diameter of the isolated afferent arteriole extracted from WKYs is about $20 \mu \mathrm{m}$, whereas that from SHRs is about $12 \mu \mathrm{m}$ (Ito, Juncos, and Carretero 1992). As the the resistance of a blood vessel is inversely proportional to its radius to the fourth power, we have

$$
\begin{aligned}
R_{A A} & \sim \frac{1}{r^{4}} & R_{A A}^{\prime} & \sim \frac{1}{r^{\prime 4}} \\
& =\frac{1}{20^{4}} & & =\frac{1}{12^{4}} \\
& =\frac{1}{160000} . & & =\frac{1}{20736} .
\end{aligned}
$$

Let $\alpha_{m y o}$ denote the factor by which the myogenic response is elevated in SHR as compared to WKY. Then we have

$$
\begin{aligned}
\alpha_{m y o} & \leq \frac{\Sigma_{m y o}^{\prime}}{\Sigma_{m y o}} \\
& =\frac{R_{A A}^{\prime}}{R_{A A}} \\
& =7.72 .
\end{aligned}
$$

To allow a safe range for this value, we set the upper bound for the sampling range of the strength of the myogenic response to be up to ten-fold $\left(\alpha_{m y o} \leq 10\right)$.

Baseline RSNA, aldosterone secretion rate, renin secretion rate, and antidiuretic hormone secretion rate are assumed in the model to range from their nominal values up to twice the nominal value. All other parameters are kept at baseline values. To estimate the value of the perturbed parameters, we fit them to two sets of experimental data: (i) MAP dynamic response to infusion of angiotensin (Ang) II (Sullivan et al. 2010), and (ii) MAP steady state response to increased/decreased sodium intake (Brinson, Rafikova, and Sullivan 2014. Wyss, Roysommuti, et al. 1994 Wyss, Chen, et al. 1987). Additionally, we impose the constraints that the aforementioned key variables are within physiological ranges.

\section{Creating the virtual population}

We employ the methodology developed by Barish et al. in creating the virtual population (Barish et al. 2017). Briefly, this technique starts with a mathematical model that is validated by fitting its parameters to aggregate data 
from a time course experiment for multiple individuals in a sample population. To simulate genetic variation, the sample population is then amplified to create the virtual population by taking bootstrap replicates of the data. The parameters of an instance of the model are then fit to each bootstrap replicate of the dataset. Here, the model parameters that we fit are the aforementioned ones which are linked to the pathophysiology in the SHR. And the dataset from which we create bootstrap replicates is the MAP dynamic response to Ang II infusion (Sullivan et al. 2010). Moreover, in creating the virtual population, we also incorporate the following experimental data when fitting the parameters. For MAP steady state response to sodium intake, there are several different reported values (Brinson, Rafikova, and Sullivan 2014, Wyss, Roysommuti, et al. 1994, Wyss, Chen, et al. 1987). So we allow for the MAP value to fall within the range of these values instead of fitting to one particular value. And we also provide a range for the constraints on the key physiological variables based upon experimental data. A flowchart outlining this procedure is given in Figure S1.

\section{Evaluating response to antihypertensive therapies}

Ang I is converted into Ang II by the angiotensin converting enzyme at a rate $c_{A C E}$ (Eq. S66). To simulate the administration of the antihypertensive drug angiotensin converting enzyme inhibitor (ACEi), we multiply this reaction rate by $\left(1-\gamma_{A C E i}\right)$, where $\gamma_{A C E i}$ is the amount of inhibition (Eq. S93). Ang II binds to its type 1 receptor (AT1R) at a rate $c_{A T 1 R}$ (Eq. S69). To simulate the administration of the antihypertensive drug angiotensin receptor blocker (ARB), we multiply this binding rate by $\left(1-\gamma_{A R B}\right)$, where $\gamma_{A R B}$ is the amount of inhibition (Eq. S94). Administration of either drug ultimately decreases the amount of Ang II bound to AT1R, which is the one of the bioactive components of the RAS. This leads to a positive feedback to increase renin secretion rate (Eqs. (S61) and S73). Thus in order to calibrate the amount of inhibition, we fit the simulated plasma renin activity after drug administration to experimental data in SHRs for ACEis (Antonaccio et al. 1979 Bunkenburg et al. 1991; Unger et al. 1986) and ARBs (Bunkenburg et al.1991; Kohara et al. 1993; Mizuno, Niimura, et al. 1992; Mizuno, Tani, et al. 1992).

Administration of a calcium channel blocker (CCB) is modeled by its postulated effect: reduction in systemic arterial resistance and renal vascular resistance (Elliott and Ram 2011; Hayashi et al. 2007). In the renal vasculature, the dihydropyridine class of CCBs dilate the afferent arteriole much more than the efferent arteriole (Honda et al. 2001). CCBs dilate smaller arteries relatively more than larger arteries (Messing et al. 1991). To model the effects of CCBs, we reduce the resistance of the afferent arteriole by a factor of $\gamma_{C C B}$, and reduce the resistance of the systemic arteries and efferent arterioles by a factor of $(2 / 3) \gamma_{C C B}$. In order to calibrate the amount of reduction in resistance, we fit the experimental vascular resistances of different diameter arteries reported after administration of a dihydropyridine class CCB in SHRs (Messing et al. 1991). This corresponds to the arterial and renal vascular resistances in the model (Eqs. (S95), (S96), and (S97)).

Thiazide diuretics (TZDs) decrease reabsorption and increase excretion of sodium and water by inhibiting the sodium chloride cotransporter along the distal convoluted tubule of the nephron. TZDs are also hypothesized to directly increase renin secretion (Duarte and Cooper-DeHoff 2010). We model the administration of TZDs by reducing the fractional amount of sodium reabsorbed in the distal segment of the nephron by a factor of $\gamma_{T Z D_{1}}$ (Eq. (S98)), while also increasing renin secretion by a factor of $\gamma_{T Z D_{2}}$ (Eq. (S99)). In order to calibrate the amount of reduction in sodium reabsorption in the distal segment, we fit the experimental plasma sodium concentration reported after administration of hydrochlorothiazide to SHRs (Yonetani et al. 1987). And we calibrate the amount of increase in renin secretion by fitting to reports of plasma renin activity in experiments after administering hydrochlorothiazide to SHRs (Lai et al. 1982 Mougenot, Mediani, and Lechat 2005). This calibration is for the particular dose used in the experimental references. To model the dose response for the entire range of inhibition effect of TZD, the effect on renin secretion, $\gamma_{T Z D_{2}}$ is assumed to be an increasing function of the effect on the fractional amount of sodium reabsorbed in the distal segment of the nephron, $\gamma_{T Z D_{1}}$. The relationship is modeled as

$$
\gamma_{T Z D_{2}}=\frac{a_{T Z D} \times \gamma_{T Z D_{1}}}{b_{T Z D}+\gamma_{T Z D_{1}}}
$$

To simulate the long term effects of administering antihypertensive drugs, we input the particular target inhibition level in Eqs. (S93)-(S100) as aforementioned and calculate the steady state solution to the model. In Figures 3a, 3b, $4 \mathrm{a}$, and $5 \mathrm{a}$, we simulate the inhibition response curve for all target inhibition levels from 0\%-99\% for ACEi, ARB, $\mathrm{CCB}$, and TZD, respectively. 


\section{Decision trees for identifying optimal drug class}

We train a decision tree classification model to determine which drug class is optimal in reducing MAP. For each drug class, we choose an inhibition level for which there is an average of $20 \%$ reduction in MAP across the virtual population. With this fixed inhibition level for each drug class, we classify each virtual individual based upon which drug class reduces MAP the most. The features of the virtual individuals are variables that are associated with the aforementioned pathophysiology of hypertension: arterial resistance, venous resistance, afferent arteriolar resistance, renin secretion rate, aldosterone secretion rate, renal sympathetic nerve activity, antidiuretic hormone secretion rate, and strength of the myogenic response. We then train a decision tree on this data set using the pathophysiological variables as predictors for which drug class is optimal. We use five-fold cross-validation to validate the model.

\section{Software implementation}

We run all simulations in MATLAB. We use MATLAB's algebraic differential equation solver ode15s and nonlinear equation solver fsolve to solve the model system of equations for time dependent and steady state simulations, respectively. We use the pattern search global optimizer patternsearch in MATLAB to fit the parameters when creating the virtual population. And we use MATLAB's Classification Learner App to train and validate the decision tree classification model. The code generated during this study are available on GitHub (https://github.com/sameedahmed/QSP-Mod-SHR.git).

\section{Complete Model Equations and Parameters}

All model equations are from Ref. (Ahmed and Layton 2020). See references therein for references for all parameter values, variable values, and equations. See Refs. (Karaaslan et al. 2005. Hallow et al. 2014 Leete and Layton 2019. Ahmed and Layton 2020) for the derivations of all of the equations.

\section{Cardiovascular function}

$$
\begin{aligned}
P_{m f} & =\left(\frac{1}{S F_{V}} \times 7.436 \times V_{b}-30.18\right) \times \epsilon_{\text {aum }} \\
P_{r a} & =\max \left\{0.2787 e^{\left.S F_{R} \times 0.2281 \Phi_{c o}-0.9119,0\right\}}\right. \\
P_{m a} & =\Phi_{c o} \times R_{t p} \\
\Phi_{v r} & =\frac{P_{m f}-P_{r a}}{R_{v r}} \\
\Phi_{c o} & =\Phi_{v r} \\
\frac{d v a s}{d t} & =v a s_{f}-v a s_{d} \\
v a s_{f} & =\frac{11.312 \times e^{-\Phi_{c o} \times\left(S F_{R} \times 0.4714\right)}}{100} \\
\text { vas } s_{d} & =v a s \times K_{v d} \\
R_{a} & =R_{b a} \times \epsilon_{\text {aum }} \\
R_{b a} & =K_{\text {bar }} / \text { vas } \\
R_{v r} & =\left(8 R_{b v}+R_{a}\right) / 31 \\
R_{t p} & =R_{a}+R_{b v} \\
\varepsilon_{\text {aum }} & =\frac{4}{5}\left(a_{\text {chemo }}+a_{\text {baro }}\right) \\
a_{\text {chemo }} & =\frac{1}{4} a_{\text {auto }} \\
\frac{d a_{\text {baro }}}{d t} & =\frac{3}{4}\left\{\frac{d a_{\text {auto }}}{d t}-0.0000667\left(a_{\text {baro }}-1\right)\right\} \\
a_{\text {auto }} & =3.0042 e^{-0.0107 P_{m a}}
\end{aligned}
$$




\section{Renal hemodynamics}

$$
\begin{aligned}
& \Phi_{r b}=\frac{P_{m a}}{R_{r}} \\
& \Phi_{g f i l t}=P_{f} \times C_{g c f} \\
& P_{f}=P_{g h}-\left(P_{B}+P_{g o}\right) \\
& P_{g h}=P_{m a}-\Phi_{r b} \times R_{A A} \\
& R_{r}=R_{A A}+R_{E A} \\
& R_{A A}=R_{A A_{0}} \times \beta_{\text {rsna }} \times \Sigma_{t g f} \times \Sigma_{m y o} \times \Psi_{A T 1 R-A A} \times \Psi_{A T 2 R-A A} \\
& R_{E A}=R_{E A_{0}} \times \Psi_{A T 1 R-E A} \times \Psi_{A T 2 R-E A} \\
& \beta_{\text {rsna }}=\frac{2}{1+e^{-3.16(\text { rsna }-1)}} \\
& \Sigma_{t g f}=0.3408+\frac{3.449}{3.88+e^{\left(\Phi_{m d-s o d}-\Phi_{\left.m d-\operatorname{sod}_{0}\right) /\left(S F_{S} \times-0.9617\right)}\right.}} \\
& \Sigma_{m y o}=0.75+\frac{b_{m y o}}{1+3.8 e^{-0.6\left(P_{g h}-63.8\right)}} \\
& \Psi_{A T 1 R-A A}=0.8+0.2092 \times \frac{[A T 1 R]}{[A T 1 R]_{e q}}-0.0092 \div \frac{[A T 1 R]}{[A T 1 R]_{e q}} \\
& \Psi_{A T 1 R-E A}=0.925+0.0835 \frac{[A T 1 R]}{[A T 1 R]_{e q}}-0.0085 \div \frac{[A T 1 R]}{[A T 1 R]_{e q}} \\
& \Psi_{A T 2 R-A A}= \begin{cases}0.75+0.25 e^{-0.15\left(\frac{[A T 2 R]}{[A T 2 R]_{e q}}-1\right)} & \text { in females } \\
1 & \text { in males }\end{cases} \\
& \Psi_{A T 2 R-E A}= \begin{cases}0.8+0.2 e^{-0.15\left(\frac{[A T 2 R]}{[A T 2 R]_{e q}}-1\right)} & \text { in females } \\
1 & \text { in males }\end{cases}
\end{aligned}
$$

\section{Renal function}

$$
\begin{aligned}
\Phi_{\text {filsod }} & =\Phi_{\text {gfilt }} \times C_{\text {sod }} \\
\Phi_{p t-\text { sodreab }} & =\Phi_{\text {filsod }} \times \eta_{p t-\text { sodreab }} \\
\Phi_{\text {md-sod }} & =\Phi_{\text {filsod }}-\Phi_{\text {pt-sodreab }} \\
\Phi_{d t-\text { sodreab }} & =\Phi_{\text {md-sod }} \times \eta_{d t-\text { sodreab }} \\
\Phi_{d t-\text { sod }} & =\Phi_{\text {md-sod }}-\Phi_{d t-\text { sodreab }} \\
\Phi_{c d-\text { sodreab }} & =\Phi_{d t-\text { sod }} \times \eta_{c d-\text { sodreab }} \\
\Phi_{u-\text { sod }} & =\Phi_{d t-\text { sod }}-\Phi_{c d-\text { sodreab }} \\
\eta_{p t-\text { sodreab }} & =\eta_{p t-\text { sodreab }}^{\text {eq }} \times \gamma_{\text {filsod }} \times \gamma_{A T 1 R} \times \gamma_{r s n a} \\
\eta_{d t-\text { sodreab }} & =\eta_{d t-\text { sodreab }}^{\text {eq }} \times \psi_{\text {al }} \\
\eta_{c d-\text { sodreab } 0} & =\eta_{c d-\text { sodreab }}^{e q} \times \lambda_{d t} \times \lambda_{\text {anp }} \times \lambda_{a l} \\
\gamma_{\text {filsod }} & =0.8+\frac{0.3}{1+e^{\left[\left(\Phi_{\text {filsod }}-\Phi_{\text {filsod }}\right) /\left(S F_{S} \times 138\right)\right]}} \\
\gamma_{A T 1 R} & =0.92+\frac{0.136}{1+e^{-1.7983\left(\frac{[A T 1 R]}{[A T 1 R]_{e q}}-0.8017\right)}} \\
\gamma_{r s n a} & =0.72+\frac{0.56}{1+e^{(1-r s n a) / 2.18}}
\end{aligned}
$$




$$
\begin{aligned}
& \psi_{a l}=\frac{11.55}{1+0.1 e^{-0.0081 C_{a l}}}-10.5 \\
& \lambda_{d t}=0.8+\frac{0.2750}{1+e^{\frac{1}{S F_{S}} \times 2.314\left(\Phi_{d t-s o d}-\Phi_{d t-\text { sod }_{0}}\right)}} \\
& \lambda_{a n p}=-0.1 \times \hat{C}_{a n p}+1.1 \\
& \lambda_{a l}=\frac{1}{C_{a l_{0}}^{0.06}} C_{a l}^{0.06} \\
& \Phi_{\text {pt-wreab }}=\Phi_{\text {gfilt }} \times \eta_{p t-w r e a b} \\
& \Phi_{m d-u}=\Phi_{\text {gfilt }}-\Phi_{p t-w r e a b} \\
& \Phi_{d t-w r e a b}=\Phi_{m d-u} \times \eta_{d t-w r e a b} \\
& \Phi_{d t-u}=\Phi_{m d-u}-\Phi_{d t-w r e a b} \\
& \Phi_{c d-w r e a b}=\Phi_{d t-u} \times \eta_{c d-w r e a b} \\
& \Phi_{u}=\Phi_{d t-u}-\Phi_{c d-w r e a b} \\
& \eta_{p t-w r e a b}=\eta_{p t-w r e a b}^{e q} \times \mu_{p t-s o d r e a b} \\
& \eta_{d t-w r e a b}=\eta_{d t-w r e a b}^{e q} \times \mu_{d t-\text { sodreab }} \\
& \eta_{c d-w r e a b}=\eta_{c d-w r e a b}^{e q} \times \mu_{c d-s o d r e a b} \times \mu_{a d h} \\
& \mu_{p t-\text { sodreab }}=0.12 \tanh \left(10\left(\frac{\eta_{p t-\text { sodreab }}}{\eta_{p t-\text { sodreab }}^{e q}}-1\right)\right)+1 \\
& \mu_{d t-\text { sodreab }}=0.12 \tanh \left(10\left(\frac{\eta_{d t-\text { sodreab }}}{\eta_{d t-\text { sodreab }}^{e q}}-1\right)\right)+1 \\
& \mu_{c d-\text { sodreab }}=0.12 \tanh \left(10\left(\frac{\eta_{c d-\text { sodreab }}}{\eta_{c d-\text { sodreab }}^{e q}}-1\right)\right)+1 \\
& \mu_{a d h}=1.0328-0.1938 e^{-0.4441 C_{a d h}}
\end{aligned}
$$

\section{Renin-angiotensin-aldosterone system}

$$
\begin{aligned}
& R_{s e c}=N_{r s} \times \nu_{m d-s o d} \times \nu_{r s n a} \times \nu_{A T 1 R} \\
& \frac{d[P R C]}{d t}=R_{\text {sec }}-\frac{\ln (2)}{h_{\text {renin }}}[P R C] \\
& P R A=[P R C] \times X_{P R C-P R A} \\
& \frac{d[A G T]}{d t}=k_{A G T}-P R A-\frac{\ln (2)}{h_{A G T}}[A G T] \\
& \frac{d[A n g I]}{d t}=P R A-\left(c_{A C E}+c_{C h y m}+c_{N E P}\right)[A n g I]-\frac{\ln (2)}{h_{A n g I}}[A n g I] \\
& \frac{d[A n g I I]}{d t}=\left(c_{A C E}+c_{C h y m}\right)[A n g I]-\left(c_{A C E 2}+c_{A I I=A I V}+c_{A T 1 R}+c_{A T 2 R}\right)[A n g I I] \\
& -\frac{\ln (2)}{h_{A n g I I}}[\text { AngII] } \\
& \frac{d[A n g(1-7)]}{d t}=c_{N E P}[A n g I]+c_{A C E 2}[A n g I I]-\frac{\ln (2)}{h_{A n g(1-7)}}[\operatorname{Ang}(1-7)] \\
& \frac{d[A n g I V]}{d t}=c_{A I I=A I V}[A n g I I]-\frac{\ln (2)}{h_{A n g I V}}[A n g I V] \\
& \frac{d[A T 1 R]}{d t}=c_{A T 1 R}[A n g I I]-\frac{\ln (2)}{h_{A T 1 R}}[A T 1 R] \\
& \frac{d[A T 2 R]}{d t}=c_{A T 2 R}[A n g I I]-\frac{\ln (2)}{h_{A T 2 R}}[A T 2 R]
\end{aligned}
$$




$$
\begin{aligned}
\nu_{m d-s o d} & =0.2262+\frac{28.04}{11.56+e^{\left(\Phi_{m d-s o d}-\Phi_{m d-s o d_{0}}\right) /\left(S F_{S} \times 0.6056\right)}} \\
\nu_{r s n a} & =1.822-\frac{2.056}{1.358+e^{(r s n a-0.8662)}} \\
\nu_{A T 1 R} & =\left(\frac{[A T 1 R]}{[A T 1 R]_{e q}}\right)^{-0.95} \\
N_{a l s} & =N_{a l s_{0}} \times \xi_{k / s o d} \times \xi_{m a p} \times \xi_{A T 1 R} \\
\frac{d N_{a l}}{d t} & =\frac{1}{T_{a l}\left(N_{a l s}-N_{a l}\right)} \\
C_{a l} & =387 N_{a l} \\
\xi_{k / \text { sod }} & =\frac{5}{1+e^{0.265\left(C_{k} / C_{s o d}-23.7\right)}} \\
\xi_{\text {map }} & = \begin{cases}70.1054 e^{-(0.0425) \times P_{m a}} & \text { if } P_{m a} \leq 100 \\
1 & \text { if } P_{m a}>100\end{cases} \\
\xi_{A T 1 R} & =0.1+\frac{2.9}{1+e^{-2\left(\frac{[A T 1 R]}{[A T 1 R]_{e q}}-1.399\right)}}
\end{aligned}
$$

\section{Miscellaneous}

$$
\begin{aligned}
& r s n a_{0}=N_{\text {rsna }} \times \alpha_{\text {map }} \times \alpha_{\text {rap }} \\
& r s n a= \begin{cases}r s n a_{0}^{\frac{1}{r s n a_{0}}} & \text { in females } \\
r s n a_{0} & \text { in males }\end{cases} \\
& \alpha_{m a p}=0.5+\frac{1}{1+e^{\left(P_{m a}-103\right) / 15}} \\
& \alpha_{\text {rap }}=1-0.008 P_{\text {ra }} \\
& \Phi_{\text {win }}=\frac{S F_{U} \times 0.002313}{1+e^{-0.8\left(C_{a d h}-4.340\right)}} \\
& \frac{d V_{e c f}}{d t}=\Phi_{w i n}-\Phi_{u} \\
& V_{b}=S F_{V} \times 4.548+\frac{S F_{V} \times 2.431}{1+e^{-\left(V_{e c f}-S F_{V} \times 18.11\right) \times\left(\frac{1}{S F_{V}} \times 0.4744\right)}} \\
& N_{a d h s}=N_{a d h s_{0}}\left[\max \left\{\left(C_{\text {sod }}-C_{\text {sod }_{0}}\right), 0\right\}+\max \left\{\left(\epsilon_{a u m}-1\right), 0\right\}-\delta_{\text {ra }}\right] / 3 \\
& \frac{d N_{a d h}}{d t}=\frac{1}{T_{a d h}}\left(N_{a d h s}-N_{a d h}\right) \\
& C_{a d h}=4 N_{a d h} \\
& \frac{d \delta_{r a}}{d t}=0.2 \frac{d P_{r a}}{d t}-0.0007 \delta_{r a} \\
& \frac{d M_{\text {sod }}}{d t}=\Phi_{\text {sodin }}-\Phi_{u-\text { sod }} \\
& C_{\text {sod }}=\frac{M_{\text {sod }}}{V_{e c f}} \\
& \hat{C}_{a n p}=7.4052-\frac{6.554}{1+e^{\left(P_{r a}-3.762\right)}}
\end{aligned}
$$

\section{Antihypertensive drug effects}

$$
c_{A C E}=c_{A C E} \times\left(1-\gamma_{A C E i}\right)
$$




$$
\begin{aligned}
c_{A T 1 R} & =c_{A T 1 R} \times\left(1-\gamma_{A R B}\right) \\
R_{a} & =R_{a} \times\left(1-\frac{2}{3} \gamma_{C C B}\right) \\
R_{E A} & =R_{E A} \times\left(1-\frac{2}{3} \gamma_{C C B}\right) \\
R_{A A} & =R_{A A} \times\left(1-\gamma_{C C B}\right) \\
\eta_{d t-\text { sodreab }} & =\eta_{d t-\text { sodreab }} \times\left(1-\gamma_{T Z D_{1}}\right) \\
R_{\text {sec }} & =R_{\text {sec }} \times\left(1+\gamma_{T Z D_{2}}\right) \\
\gamma_{T Z D_{2}} & =\frac{a_{T Z D} \times \gamma_{T Z D_{1}}}{b_{T Z D}+\gamma_{T Z D_{1}}}
\end{aligned}
$$




\section{Supplemental References}

Ahmed, Sameed and Anita T. Layton (2020). "Sex-specific computational models for blood pressure regulation in the rat". In: American Journal of Physiology-Renal Physiology 318.4. Publisher: American Physiological Society, F888-F900. DOI: 10.1152/ajprenal.00376.2019.

Antonaccio, Michael J. et al. (1979). "Effects of Chronic Treatment with Captopril (SQ 14, 225), an Orally Active Inhibitor of Angiotensin I-Converting Enzyme, in Spontaneously Hypertensive Rats". In: The Japanese Journal of Pharmacology 29.2, pp. 285-294. DOI: 10.1254/jjp.29.285

Arendshorst, W. J. and W. H. Beierwaltes (1979). "Renal and nephron hemodynamics in spontaneously hypertensive rats". In: American Journal of Physiology-Renal Physiology 236.3, F246-F251. DOI: 10.1152/ajprenal.1979. 236.3.F246.

Badyal, D. K., A. P. Dadhich, and H. Lata (2003). "Animal models of hypertension and effect of drugs". In: Indian Journal of Pharmacology 35.6. Company: Indian Journal of Pharmacology Distributor: Indian Journal of Pharmacology Institution: Indian Journal of Pharmacology Label: Indian Journal of Pharmacology Publisher: Medknow Publications, p. 349.

Barish, Syndi et al. (2017). "Evaluating optimal therapy robustness by virtual expansion of a sample population, with a case study in cancer immunotherapy". In: Proceedings of the National Academy of Sciences 114.31, E6277E6286. DOI: 10.1073/pnas.1703355114.

Beierwaltes, W H, W J Arendshorst, and P J Klemmer (1982). "Electrolyte and water balance in young spontaneously hypertensive rats." In: Hypertension 4.6, pp. 908-915. DOI: 10.1161/01.HYP.4.6.908.

Bianchi, G. et al. (1974). "Blood Pressure Changes Produced by Kidney Cross-Transplantation between Spontaneously Hypertensive Rats and Normotensive Rats". In: Clinical Science 47.5, pp. 435-448. DoI: $10.1042 /$ cs0470435.

Brinson, Krystal N., Olga Rafikova, and Jennifer C. Sullivan (2014). "Female sex hormones protect against saltsensitive hypertension but not essential hypertension". In: American Journal of Physiology-Regulatory, Integrative and Comparative Physiology 307.2, R149-R157. DOI: 10.1152/ajpregu.00061.2014.

Bunkenburg, B et al. (1991). "Prolonged angiotensin II antagonism in spontaneously hypertensive rats. Hemodynamic and biochemical consequences." In: Hypertension 18.3, pp. 278-288. DOI: 10.1161/01.HYP.18.3.278

Crofton, J. T. et al. (1978). "Vasopressin in the rat with spontaneous hypertension". In: American Journal of Physiology-Heart and Circulatory Physiology 235.4, H361-H366. DOI: 10.1152/ajpheart.1978.235.4.H361.

Duarte, Julio D and Rhonda M Cooper-DeHoff (2010). "Mechanisms for blood pressure lowering and metabolic effects of thiazide and thiazide-like diuretics". In: Expert Review of Cardiovascular Therapy 8.6, pp. 793-802. DOI: $10.1586 /$ erc.10.27.

Elliott, William J. and C. Venkata S. Ram (2011). "Calcium Channel Blockers: Calcium Channel Blockers". In: The Journal of Clinical Hypertension 13.9, pp. 687-689. DOI: 10.1111/j.1751-7176.2011.00513.x.

Freeman, R H et al. (1975). "Control of aldosterone secretion in the spontaneously hypertensive rat." In: Circulation Research 37.1, pp. 66-71. DOI: 10.1161/01.RES.37.1.66.

Hallow, K. Melissa et al. (2014). "A model-based approach to investigating the pathophysiological mechanisms of hypertension and response to antihypertensive therapies: extending the Guyton model". In: American Journal of Physiology-Regulatory, Integrative and Comparative Physiology 306.9, R647-R662. DOI: 10.1152/ajpregu.00039. 2013.

Hayashi, Koichi et al. (2007). "Ca ${ }^{2+}$ Channel Subtypes and Pharmacology in the Kidney". In: Circulation Research 100.3, pp. 342-353. DOI: 10.1161/01.RES.0000256155.31133.49.

Honda, Masanori et al. (2001). "Divergent renal vasodilator action of L- and T-type calcium antagonists in vivo:" in: Journal of Hypertension 19.11, pp. 2031-2037. DOI: 10.1097/00004872-200111000-00014.

Ito, S., L. A. Juncos, and O. A. Carretero (1992). "Pressure-induced constriction of the afferent arteriole of spontaneously hypertensive rats." In: Hypertension 19.2, pp. II164-II164. DOI: 10.1161/01.HYP.19.2_Suppl.II164.

Judy, W V and S K Farrell (1979). "Arterial baroreceptor reflex control of sympathetic nerve activity in the spontaneously hypertensive rat." In: Hypertension 1.6, pp. 605-614. DOI: 10.1161/01.HYP.1.6.605

Karaaslan, Fatih et al. (2005). "Long-Term Mathematical Model Involving Renal Sympathetic Nerve Activity, Arterial Pressure, and Sodium Excretion". In: Annals of Biomedical Engineering 33.11, pp. 1607-1630. DOI: 10.1007/ s10439-005-5976-4.

Kohara, Katsuhiko et al. (1993). "Peripheral vs. Central Blockade of the Renin- Angiotensin System in Spontaneously Hypertensive Rats: Comparison of Novel $\mathrm{AT}_{1}$ Receptor Antagonist TCV-116 with Angiotensin Converting Enzyme Inhibitor Delapril". In: Hypertension Research 16.4, pp. 239-246. DOI: 10.1291/hypres.16.239. 
Lai, F. M. et al. (1982). "Studies on the Mechanism of the Enhancement of the Antihypertensive Activity of Captopril by A Diuretic in Spontaneously Hypertensive Rats". In: Clinical and Experimental Hypertension. Part A: Theory and Practice 4.6, pp. 1001-1018. DOI: 10.3109/10641968209060768.

Lee, H. B. and M. D. Blaufox (1985). "Blood Volume in the Rat". In: Journal of Nuclear Medicine 26.1, pp. 72-76.

Leete, Jessica and Anita T. Layton (2019). "Sex-specific long-term blood pressure regulation: Modeling and analysis". In: Computers in Biology and Medicine 104, pp. 139-148. DOI: 10.1016/j.compbiomed.2018.11.002.

Liard, J. -F. (1977). "Renal denervation delays blood pressure increase in the spontaneously hypertensive rat". In: Experientia 33.3, pp. 339-340. DOI: 10.1007/BF02002815.

Messing, Marcel et al. (1991). "Microvascular actions of calcium channel antagonists". In: European Journal of Pharmacology 198.2, pp. 189-195. DOI: 10.1016/0014-2999(91)90620-6.

Mizuno, Kenji, Susumu Niimura, et al. (1992). "Antihypertensive and hormonal activity of MK 954 in spontaneously hypertensive rats". In: European Journal of Pharmacology 215.2, pp. 305-308. DOI: 10.1016/0014-2999(92) 90045-6.

Mizuno, Kenji, Makio Tani, et al. (1992). "Effects of losartan, a nonpeptide angiotensin II receptor antagonist, on cardiac hypertrophy and the tissue angiotensin II content in spontaneously hypertensive rats". In: Life Sciences 51.5, pp. 367-374. DOI: 10.1016/0024-3205(92) 90589-H.

Mougenot, N, O Mediani, and P Lechat (2005). "Bisoprolol and hydrochlorothiazide effects on cardiovascular remodeling in spontaneously hypertensive rats". In: Pharmacological Research 51.4, pp. 359-365. DOI: 10.1016/j.phrs. 2004.10.010.

Okamoto, Kozo and Kyuzo Aoki (1963). "Development of a Strain of Spontaneously Hypertensive Rats". In: Japanese Circulation Journal 27.3, pp. 282-293. DOI: 10.1253/jcj.27.282.

Peeters, H. (2014). Protides of the Biological Fluids: Proceedings of the Twentieth Colloquium, Brugge, 1972. GoogleBooks-ID: ctmNDAAAQBAJ. Elsevier. 628 pp.

Sullivan, Jennifer C. et al. (2010). "Angiotensin (1-7) Receptor Antagonism Equalizes Angiotensin II-Induced Hypertension in Male and Female Spontaneously Hypertensive Rats". In: Hypertension 56.4, pp. 658-666. DOI: 10.1161/HYPERTENSIONAHA.110.153668.

Unger, Thomas et al. (1986). "Antihypertensive Action of the Converting Enzyme Inhibitor Perindopril (S9490-3) in Spontaneously Hypertensive Rats: Comparison with Enalapril (MK421) and Ramipril (HOE498)". In: Journal of Cardiovascular Pharmacology 8.2, pp. 276-285.

Wyss, J M, Y F Chen, et al. (1987). "Spontaneously hypertensive rats exhibit reduced hypothalamic noradrenergic input after $\mathrm{NaCl}$ loading." In: Hypertension 10.3, pp. 313-320. DOI: 10.1161/01.HYP.10.3.313.

Wyss, J M, S Roysommuti, et al. (1994). "Salt-induced hypertension in normotensive spontaneously hypertensive rats." In: Hypertension 23.6, pp. 791-796. DOI: 10.1161/01.HYP. 23.6.791.

Yonetani, Yukio et al. (1987). "A new diuretic that does not reduce renal handling of uric acid in rats, S-8666." In: The Japanese Journal of Pharmacology 43.4, pp. 399-405. DOI: 10.1254/jjp.43.399. 